\title{
Threshold Dynamics in a Model for Zika Virus Disease with Seasonality
}

\author{
Mahmoud A. Ibrahim ${ }^{1,2}$ (D) Attila Dénes ${ }^{1}$
}

Received: 7 June 2020 / Accepted: 9 December 2020 / Published online: 17 February 2021

(c) The Author(s) 2021

\begin{abstract}
We present a compartmental population model for the spread of Zika virus disease including sexual and vectorial transmission as well as asymptomatic carriers. We apply a non-autonomous model with time-dependent mosquito birth, death and biting rates to integrate the impact of the periodicity of weather on the spread of Zika. We define the basic reproduction number $\mathscr{R}_{0}$ as the spectral radius of a linear integral operator and show that the global dynamics is determined by this threshold parameter: If $\mathscr{R}_{0}<1$, then the disease-free periodic solution is globally asymptotically stable, while if $\mathscr{R}_{0}>1$, then the disease persists. We show numerical examples to study what kind of parameter changes might lead to a periodic recurrence of Zika.
\end{abstract}

Keywords Periodic epidemic model · Zika virus (ZIKV) - Global stability · Uniform persistence

\section{Introduction}

Zika virus disease or Zika fever is a mosquito-borne disease caused by the Zika virus (ZIKV). This Flavivirus was first identified in monkeys in Uganda in 1947 (Dick et al. 1952), then identified in humans in 1952 in Uganda and Tanzania (Smithburne 1952). The first cases of Zika infection in South America were detected in Brazil in spring 2015 and several further countries from the region reported Zika cases in the same year. Zika virus is chiefly spread in tropical and subtropical regions by the bite of infected female mosquitoes from the Aedes genus (by Aedes aegypti above all) (see, e.g., Petersen et al. 2016), the same species that is responsible for dengue, chikungunya and yellow fever transmission. Zika virus is also spread via sexual contacts, principally from men to women (Magalhaes et al. 2018). Studies suggest that ZIKV might remain

\footnotetext{
$凶 \quad$ Mahmoud A. Ibrahim

mibrahim@math.u-szeged.hu

1 Bolyai Institute, University of Szeged, Aradi vértanúk tere 1., Szeged 6720, Hungary

2 Department of Mathematics, Faculty of Science, Mansoura University, Mansoura 35516, Egypt
} 
in male genital secretions for a longer period (up to 6 months) than in other bodily fluids, hence, in this way, a transmission of the disease is possible even several months after recovery (Mead et al. 2018). Mothers can transmit the disease to their fetus during pregnancy or during delivery. This transmission might result in microcephaly (a medical condition with improper brain development and head size smaller than normal) and further congenital malformations. These are collectively denominated as congenital Zika syndrome. The incubation period of Zika virus disease is around 3-14 days. Most of the infected people do not show any symptoms or only mild ones including fever, rash, muscle and joint pain, conjunctivitis and headache, in general lasting for 2-7 days (WHO 2015).

A number of sophisticated mathematical models for the spread of Zika virus disease have been previously developed, see e.g. Brauer et al. (2016); Padmanabhan et al. (2017); Baca-Carrasco and Velasco-Hernández (2016). Gao et al. (2016) presented an autonomous compartmental model of Zika spread considering mosquito-borne and sexual transmission proposing an SEIR-type model for the human population with $S, E$ and $I$ compartments for vectors. They separated asymptomatically infected humans from those who had symptoms. Sasmal et al. (2018) established a stagestructured model to study the effect of sexual transmission. Caminade et al. (2016) and Mordecai et al. (2017) formulated a compartmental model of Zika transmission which considers the importance of weather and climate changes. In Dénes et al. (2019) a non-autonomous model was established considering most of the important features regarding Zika transmission: sexual and vector-borne transmission, the role of asymptomatically infected humans, the prolonged period of infectiousness after recovery and assessed the importance of the seasonality of weather. In (Ibrahim and Dénes 2019), this model was extended to improve the estimation of microcephaly risk due to Zika. However, most models so far have not considered seasonality, although the number of mosquitoes - and thus the number of infections - is highly dependent on the periodically changing weather circumstances. Hence, in the present work, we establish and study a model with nine compartments describing the spread of Zika virus disease in a periodically changing environment: we set the mosquito birth and death rates as well as the biting rates to be periodic with 1 year as period, following the annual change of weather. The study of such models was initiated and further extended in (Bacaër and Guernaoui 2006; Wang and Zhao 2008; Rebelo et al. 2011; Bacaër and Ait Dads 2011), where a general definition was introduced for the basic reproduction number of periodic compartmental models, defined as the spectral radius of an integral operator acting on the space of continuous periodic functions and the reproduction number was also shown to be a threshold parameter for the local stability of the disease-free periodic solution. Since then, several papers have used the methods introduced in the above works; see, e.g., (Bakary et al. 2018; Zhang and Zhao 2007; Wang et al. 2013; Liu et al. 2010; Nakata and Kuniya 2010).

Our aim is to determine the basic reproduction number for our newly established periodic model which serves as a threshold parameter regarding the persistence of the disease. In the analysis we follow the methods established in the above-cited papers, however, the techniques need to be adapted to the present model including both humanhuman and mosquito-human transmission. Further, it is an utmost important question to know what might lead to a regular recurrence of the epidemic. Several vector- 
borne diseases - malaria, dengue, chikungunya — tend to reappear from year to year, following the annual periodicity of weather. Up to now, unlike these diseases, after 1-3 major outbreaks in following years in various countries, Zika has not shown a periodic recurrence. Our hope is that our model might help to understand which changes in the parameters may contribute to such a phenomenon. This is especially important in the days of climate change, which might provoke important modifications in the mosquito-related parameters. Furthermore, other factors like mutations of the virus might also change sexual transmission rates as well.

The paper is organized as follows. In Sect. 2, we introduce our periodic compartmental model for Zika fever transmission. In Sect. 3, we determine the basic reproduction number and study the local asymptotic stability of the disease-free periodic solution. In Sect. 4, we study the global stability of the disease-free equilibrium in the case of $\mathscr{R}_{0}<1$ the persistence of the disease in case of $\mathscr{R}_{0}>1$. We also calculate the basic reproduction number of the time-constant variant of the model. In Sect. 5, we present a case study for two South American countries. We estimate the parameter values for both countries and perform sensitivity analysis to determine the parameters which have the largest effect on the outcome of the epidemic. We provide numerical simulations to study the possible effects of an alteration of various parameters to see what kind of changes might lead to an annual recurrence of the disease. The paper is closed by a discussion.

\section{Mathematical Model}

We divide the total human population into six compartments: susceptible $S_{h}(t)$, exposed $E_{h}(t)$, symptomatically infected $I_{S}(t)$, asymptomatically infected $I_{a}(t)$, convalescent $I_{r}(t)$, and recovered $R(t)$ at time $t>0$, while the vector population is divided into three classes: susceptible $S_{v}(t)$, exposed $E_{v}(t)$, and infectious $I_{v}(t)$ individuals.

The total human population $N_{h}(t)$ and the total mosquito population $N_{v}(t)$ are given by:

$$
\begin{aligned}
& N_{h}(t)=S_{h}(t)+E_{h}(t)+I_{a}(t)+I_{S}(t)+I_{r}(t)+R(t), \\
& N_{v}(t)=S_{v}(t)+E_{v}(t)+I_{v}(t) .
\end{aligned}
$$

Our model takes the form

$$
\begin{aligned}
S_{h}^{\prime}(t)= & \mu_{h}-\beta \frac{\tau_{e} E_{h}(t)+\tau_{a} I_{a}(t)+I_{s}(t)+\tau_{r} I_{r}(t)}{N_{h}(t)} S_{h}(t)-d_{h} S_{h}(t) \\
& -\frac{\tilde{\alpha}_{h}(t)}{N_{h}(t)} I_{v}(t) S_{h}(t), \\
E_{h}^{\prime}(t)= & \beta \frac{\tau_{e} E_{h}(t)+\tau_{a} I_{a}(t)+I_{s}(t)+\tau_{r} I_{r}(t)}{N_{h}(t)} S_{h}(t)+\frac{\tilde{\alpha}_{h}(t)}{N_{h}(t)} I_{v}(t) S_{h}(t) \\
& -v_{h} E_{h}(t)-d_{h} E_{h}(t), \\
I_{a}^{\prime}(t)= & q v_{h} E_{h}(t)-\gamma_{a} I_{a}(t)-d_{h} I_{a}(t), \\
I_{s}^{\prime}(t)= & (1-q) v_{h} E_{h}(t)-\gamma_{s} I_{S}(t)-d_{h} I_{s}(t),
\end{aligned}
$$




$$
\begin{aligned}
I_{r}^{\prime}(t) & =\gamma_{a} I_{a}(t)+\gamma_{s} I_{s}(t)-\gamma_{r} I_{r}(t)-d_{h} I_{r}(t), \\
R^{\prime}(t) & =\gamma_{r} I_{r}(t)-d_{h} R(t), \\
S_{v}^{\prime}(t) & =\tilde{\mu}_{v}(t)-\tilde{\alpha}_{v}(t) \frac{\eta_{e} E_{h}(t)+\eta_{a} I_{a}(t)+I_{s}(t)}{N_{h}(t)} S_{v}(t)-\tilde{d}_{v}(t) S_{v}(t), \\
E_{v}^{\prime}(t) & =\tilde{\alpha}_{v}(t) \frac{\eta_{e} E_{h}(t)+\eta_{a} I_{a}(t)+I_{s}(t)}{N_{h}(t)} S_{v}(t)-v_{v} E_{v}(t)-\tilde{d}_{v}(t) E_{v}(t), \\
I_{v}^{\prime}(t) & =v_{v} E_{v}(t)-\tilde{d}_{v}(t) I_{v}(t),
\end{aligned}
$$

where $\tilde{\mu}_{v}(t), \tilde{\alpha}_{h}(t), \tilde{\alpha}_{v}(t)$ and $\tilde{d}_{v}(t)$ denote mosquito birth rate, transmission rate from an infectious mosquito to a susceptible human, the transmission rate from infected humans to susceptible mosquitoes and mosquito death rate, respectively. In our model, we assumed $\tilde{\mu}_{v}(t), \tilde{\alpha}_{h}(t), \tilde{\alpha}_{v}(t)$ and $\tilde{d}_{v}(t)$ to be continuous, positive $\omega$ periodic functions. An individual may progress from susceptible $\left(S_{h}\right)$ to exposed $\left(E_{h}\right)$ upon contracting the disease. An exposed individual moves either to the symptomatically infected class $I_{s}$ or to the asymptomatically infected class $I_{a}$, depending on whether that person shows symptoms or not. Infected people with or without symptoms move to the convalescent compartment $I_{r}$ including those who have already recovered, but who can still transmit the disease via sexual contact. After the convalescent period, one moves to the recovered compartment $R$. Mosquitoes may progress from susceptible $\left(S_{v}\right)$ to exposed $\left(E_{v}\right)$ and then to infectious $\left(I_{v}\right)$ class. The description of the model parameters is summarized in Table 1 , while the transmission diagram of the model can be seen in Fig. 1. We note that although the population is non-constant, the recruitment term in our model is given as $\mu_{h}$ instead of $\mu_{h} N_{h}$, as the countries studied in this work can be expected to be close to constant within a reasonable time interval. Doing so, we also followed among others the works Bacaër and Guernaoui (2006), Liu et al. (2010), Qu et al. (2017), Wang et al. (2013). We emphasize that a similar model was established and studied in Dénes et al. (2019), which also included differentiation of the two sexes. However, no stability analysis was performed in that paper, only numerical results were presented.

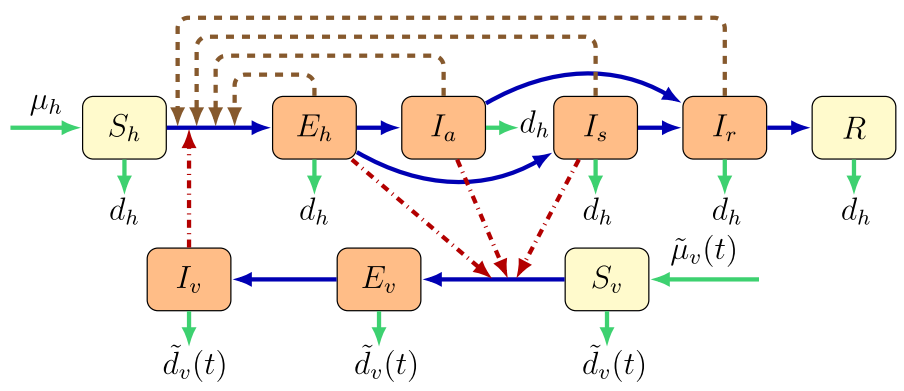

Fig. 1 (Color figure online) Zika virus dynamics spread including vectorial and sexual transmission. Brown nodes are infectious and yellow nodes are non-infectious. Blue solid arrows show the progression of infection, while brown dashed arrows show direction of human-to-human transmission and red dash-dotted arrows show direction of transmission between humans and mosquitoes. Green arrows show birth and death 
Table 1 Description of parameters of model 1

\begin{tabular}{ll}
\hline Parameter & Description \\
\hline$\mu_{h}$ & Human birth rate \\
$d_{h}$ & Human death rate \\
$\beta$ & Transmission rate from infected humans to susceptible humans \\
$\alpha_{h}$ & Baseline value of transmission rate from mosquitoes to humans \\
$\alpha_{v}$ & Baseline value of transmission rate from humans to mosquitoes \\
$q$ & Proportion of asymptomatic infections \\
$\tau_{e}, \tau_{a}, \tau_{r}$ & Relative human-to-human transmissibility of (exposed, asymptomatic and \\
$\eta_{e}, \eta_{a}$ & convalescent) humans to symptomatic humans \\
$\gamma_{a}$ & Relative human-to-mosquito transmissibility of (exposed and asymptomatically \\
$\gamma_{s}$ & infected) humans to symptomatically infected humans \\
$\gamma_{r}$ & Progression rate from $I_{a}$ to $I_{r}$ \\
$v_{h}$ & Progression rate from $I_{s}$ to $I_{r}$ \\
$v_{v}$ & Recovery rate of convalescent humans \\
$\mu_{v}$ & Human incubation rate \\
$d_{v}$ & Mosquitoes incubation rate \\
\hline & Baseline value of mosquito birth rate \\
& Baseline value of mosquito death rate
\end{tabular}

We define $X_{h}=\left(S_{h}, E_{h}, I_{a}, I_{s}, I_{r}, R\right)$ and the functions $g_{1}, g_{2}, g_{3} \in C\left(\mathbb{R}_{+}^{6}, \mathbb{R}_{+}\right)$ by

$$
\begin{aligned}
& g_{1}\left(X_{h}\right)= \begin{cases}0, & \text { if } X_{h}=(0,0,0,0,0,0), \\
\frac{\tau_{e} E_{h}+\tau_{a} I_{a}+I_{s}+\tau_{r} I_{r}}{S_{h}+E_{h}+I_{a}+I_{s}+I_{r}+R} S_{h}, & \text { if } X_{h} \in \mathbb{R}_{+}^{6} \backslash\{(0,0,0,0,0,0)\},\end{cases} \\
& g_{2}\left(X_{h}\right)= \begin{cases}0, & \text { if } X_{h}=(0,0,0,0,0,0), \\
\frac{1}{S_{h}+E_{h}+I_{a}+I_{s}+I_{r}+R} S_{h}, & \text { if } X_{h} \in \mathbb{R}_{+}^{6} \backslash\{(0,0,0,0,0,0)\},\end{cases} \\
& g_{3}\left(X_{h}\right)= \begin{cases}0, & \text { if } X_{h}=(0,0,0,0,0,0), \\
\frac{\eta_{e} E_{h}(t)+\eta_{a} I_{a}(t)+I_{s}(t)}{S_{h}+E_{h}+I_{a}+I_{s}+I_{r}+R}, & \text { if } X_{h} \in \mathbb{R}_{+}^{6} \backslash\{(0,0,0,0,0,0)\}\end{cases}
\end{aligned}
$$

Clearly, $g_{1}\left(X_{h}\right), g_{2}\left(X_{h}\right)$ and $g_{3}\left(X_{h}\right)$ are continuous on $\mathbb{R}_{+}^{6}$. Also, $g_{1}\left(X_{h}\right), g_{2}\left(X_{h}\right)$ and $g_{3}\left(X_{h}\right)$ are globally Lipschitz on $\mathbb{R}_{+}^{6}$. By a change of variable $N_{h}=S_{h}+E_{h}+I_{a}+$ $I_{s}+I_{r}+R$ and from (2), system (1) is equivalent to

$$
\begin{aligned}
S_{h}^{\prime}(t)= & \mu_{h}-\beta g_{1}\left(S_{h}, E_{h}, I_{a}, I_{s}, I_{r}, N_{h}\right)-\tilde{\alpha}_{h}(t) g_{2}\left(S_{h}, E_{h}, I_{a}, I_{s}, I_{r}, N_{h}\right) I_{v}(t) \\
& -d_{h} S_{h}(t), \\
E_{h}^{\prime}(t)= & \beta g_{1}\left(S_{h}, E_{h}, I_{a}, I_{s}, I_{r}, N_{h}\right)+\tilde{\alpha}_{h}(t) g_{2}\left(S_{h}, E_{h}, I_{a}, I_{s}, I_{r}, N_{h}\right) I_{v}(t) \\
& -v_{h} E_{h}(t)-d_{h} E_{h}(t), \\
I_{a}^{\prime}(t)= & q v_{h} E_{h}(t)-\gamma_{a} I_{a}(t)-d_{h} I_{a}(t), \\
I_{s}^{\prime}(t)= & (1-q) v_{h} E_{h}(t)-\gamma_{s} I_{s}(t)-d_{h} I_{s}(t),
\end{aligned}
$$




$$
\begin{aligned}
& I_{r}^{\prime}(t)=\gamma_{a} I_{a}(t)+\gamma_{s} I_{s}(t)-\gamma_{r} I_{r}(t)-d_{h} I_{r}(t) \\
& N_{h}^{\prime}(t)=\mu_{h}-d_{h} N_{h}(t) \\
& S_{v}^{\prime}(t)=\tilde{\mu}_{v}(t)-\tilde{\alpha}_{v}(t) g_{3}\left(S_{h}, E_{h}, I_{a}, I_{s}, I_{r}, N_{h}\right) S_{v}(t)-\tilde{d}_{v}(t) S_{v}(t) \\
& E_{v}^{\prime}(t)=\tilde{\alpha}_{v}(t) g_{3}\left(S_{h}, E_{h}, I_{a}, I_{s}, I_{r}, N_{h}\right) S_{v}(t)-v_{v} E_{v}(t)-\tilde{d}_{v}(t) E_{v}(t), \\
& I_{v}^{\prime}(t)=v_{v} E_{v}(t)-\tilde{d}_{v}(t) I_{v}(t)
\end{aligned}
$$

We now prove the existence of a disease-free periodic solution of (3). For the human subsystem of system (3) with initial condition

$$
X^{0}=\left(S_{h}(0), E_{h}(0), I_{a}(0), I_{s}(0), I_{r}(0), N_{h}(0), S_{v}(0), E_{v}(0), I_{v}(0)\right) \in \mathbb{R}_{+}^{9},
$$

we have the linear differential equation

$$
\frac{\mathrm{d} N_{h}}{\mathrm{~d} t}(t)=\mu_{h}-d_{h} N_{h}(t)
$$

One can easily see that (4) has a single equilibrium $N_{h}^{*}=\frac{\mu_{h}}{d_{h}}$, which is globally asymptotically stable and $N_{h}(t)$ is bounded.

To determine the disease-free periodic solution of (3), we study equation

$$
S_{v}^{\prime}(t)=\tilde{\mu}_{v}(t)-\tilde{d}_{v}(t) S_{v}(t)
$$

with initial value $S_{v}(0) \in \mathbb{R}_{+}$. Equation (5) has a single positive $\omega$-periodic solution $S_{v}^{*}(t)$, globally attractive in $\mathbb{R}_{+}$and thus, system (3) has a single disease-free periodic solution $E_{0}=\left(N_{h}^{*}, 0,0,0,0, N_{h}^{*}, S_{v}^{*}(t), 0,0\right)$.

To formulate our next result, we introduce the notations $h^{L}=\sup _{t \in[0, \omega)} h(t)$ and $h^{M}=\inf _{t \in[0, \omega)} h(t)$ for a continuous, positive $\omega$-periodic function $h(t)$.

Lemma 1 There exists an $N_{v}^{*}=\frac{\tilde{\mu}_{v}^{L}}{\tilde{d}_{v}^{L}}>0$ such that every forward solution in $X:=$ $\left\{\left(S_{h}, E_{h}, I_{a}, I_{s}, I_{r}, N_{h}, S_{v}, E_{v}, I_{v}\right) \in \mathbb{R}_{+}^{9}: N_{h} \geq S_{h}+E_{h}+I_{a}+I_{s}+I_{r}, N_{v}\right.$ $\left.\geq S_{v}+E_{v}+I_{v}\right\}$ of (3) eventually enters

$$
\begin{aligned}
& G_{N^{*}}:=\left(S_{h}, E_{h}, I_{a}, I_{s}, I_{r}, N_{h}, S_{v}, E_{v}, I_{v}\right) \in X: N_{h} \\
&\left.\leq N_{h}^{*}, S_{v}+E_{v}+I_{v} \leq N_{v}^{*}<\infty\right\}
\end{aligned}
$$

and for each $N_{v}(t) \geq N_{v}^{*}, G_{N}$ is positively invariant for (3). Further, it holds that

$$
\lim _{t \rightarrow+\infty}\left(N_{v}(t)-S_{v}^{*}(t)\right)=0
$$

where $N_{v}(t)=S_{v}(t)+E_{v}(t)+I_{v}(t)$.

Proof From (3), for the mosquito subsystem, we have

$$
N_{v}^{\prime}(t)=\tilde{\mu}_{v}(t)-\tilde{d}_{v}(t) N_{v}(t) \leq \mu_{v}^{L}-d_{v}^{M} N_{v}(t) \leq 0, \quad \text { if } N_{v}(t) \geq N_{v}^{*}
$$


which implies that $G_{N}, N_{v}(t) \geq N_{v}^{*}$, is forward invariant and eventually, every positive orbit will enter $G_{N^{*}}$. For the second part of the proof, let us assume that

$$
y(t)=N_{v}(t)-S_{v}^{*}(t), \quad t \geq 0 .
$$

We then have that

$$
y^{\prime}(t)=-\tilde{d}_{v}(t) y(t)
$$

which implies that

$$
\lim _{t \rightarrow+\infty} y(t)=0
$$

Hence, the proof is complete.

\section{Basic Reproduction Number, Local Stability}

Following the technique and the notations introduced by Wang and Zhao (2008), we show the local stability of the disease-free periodic equilibrium $E_{0}$ of (3) for appropriate parameter values. First, we introduce the basic reproduction number $\mathscr{R}_{0}$ for system (3). Let $\mathscr{X}=\left(E_{h}, I_{a}, I_{s}, I_{r}, E_{v}, I_{v}, S_{h}, N_{h}, S_{v}\right)^{T}$ with $\mathscr{F}(t, \mathscr{X}(t)), \mathscr{V}^{+}(t, \mathscr{X}(t))$ and $\mathscr{V}^{-}(t, \mathscr{X}(t))$ denote the input rate of newly infected individuals, the input rate of individuals by other means and the rate of transfer of individuals out of compartments, respectively.

System (3) is equivalent to

$$
\mathscr{X}^{\prime}(t)=\mathscr{F}(t, \mathscr{X}(t))-\mathscr{V}(t, \mathscr{X}(t)),
$$

where $\mathscr{V}(t, \mathscr{X}(t))=\mathscr{V}^{-}(t, \mathscr{X}(t))-\mathscr{V}^{+}(t, \mathscr{X}(t))$. We know that (6) has the diseasefree periodic solution $\mathscr{X}^{*}(t)=\left(0,0,0,0,0,0, N_{h}^{*}, N_{h}^{*}, S_{v}^{*}(t)\right)$. Then by a simple computation we obtain

$$
\begin{aligned}
F(t)= & {\left[\begin{array}{cccccc}
\beta \tau_{e} & \beta \tau_{a} & \beta & \beta \tau_{r} & 0 & \tilde{\alpha}_{h}(t) \\
0 & 0 & 0 & 0 & 0 & 0 \\
0 & 0 & 0 & 0 & 0 & 0 \\
0 & 0 & 0 & 0 & 0 & 0 \\
\frac{\eta_{e} \tilde{\alpha}_{v}(t)}{N_{h}^{*}} S_{v}^{*}(t) & \frac{\eta_{a} \tilde{\alpha}_{v}(t)}{N_{h}^{*}} S_{v}^{*}(t) & \frac{\tilde{\alpha}_{v}(t)}{N_{h}^{*}} S_{v}^{*}(t) & 0 & 0 & 0 \\
0 & 0 & 0 & 0 & 0 & 0
\end{array}\right], } \\
V(t)= & {\left[\begin{array}{ccccccc}
v_{h}+d_{h} & 0 & 0 & 0 & 0 & 0 \\
-q v_{h} & \gamma_{a}+d_{h} & 0 & 0 & 0 & 0 \\
-(1-q) \nu_{h} & 0 & \gamma_{s}+d_{h} & 0 & 0 & 0 \\
0 & -\gamma_{a} & -\gamma_{s} & \gamma_{r}+d_{h} & 0 & 0 \\
0 & 0 & 0 & 0 & v_{v}+\tilde{d}_{v}(t) & 0 \\
0 & 0 & 0 & 0 & -v_{v} & \tilde{d}_{v}(t)
\end{array}\right], }
\end{aligned}
$$




$$
M(t)=\left[\begin{array}{ccc}
-d_{h} & 0 & 0 \\
0 & -d_{h} & 0 \\
0 & 0 & -\tilde{d}_{v}(t)
\end{array}\right]
$$

Furthermore, $F(t)$ is non-negative, and $-V(t)$ is cooperative. Also $F(t)-V(t)$ is irreducible for all $t$. It is straightforward to see that the conditions (A1)-(A6) are satisfied, and $\mathscr{X}^{*}(t)$ is linearly asymptotically stable in the disease-free subspace

$$
\mathscr{X}_{s}=\left(0,0,0,0,0,0, S_{h}, N_{h}, S_{v}\right) \in \mathbb{R}_{+}^{9} .
$$

Assume $Y(t, s), t \geq s$ is the evolution operator of the linear $\omega$-periodic system

$$
\frac{\mathrm{d} y}{\mathrm{~d} t}=-V(t) y
$$

That is, for each $s \in \mathbb{R}$, the $6 \times 6$ matrix $Y(t, s)$ satisfies

$$
\frac{\mathrm{d}}{\mathrm{d} t} Y(t, s)=-V(t) Y(t, s), \quad \forall t \geq s, Y(s, s)=I,
$$

where $I$ is the $6 \times 6$ identity matrix. Thus, the monodromy matrix $\Phi_{-V}(t)$ of (8) is equal to $Y(t, 0), t \geq 0$. Therefore, the condition (A7) holds.

Suppose $\phi(s), \omega$-periodic in $s$, is the initial distribution of infectious individuals. Then $F(s) \phi(s)$ gives us the rate of new infections produced by the infected individuals introduced at time $s$. Given $t \geq s$, then $Y(t, s) F(s) \phi(s)$ supplies the distribution of those newly infected at time $s$ who remain in the infected compartments at time $t$. Then

$$
\psi(t):=\int_{-\infty}^{t} Y(t, s) F(s) \phi(s) \mathrm{d} s=\int_{0}^{\infty} Y(t, t-a) F(t-a) \phi(t-a) d a,
$$

is the distribution of accumulative new infections at time $t$ due to all infected individuals $\phi(s)$ introduced at time less than $t$.

We denote by $C_{\omega}$ the ordered Banach space of $\omega$-periodic functions from $\mathbb{R}$ to $\mathbb{R}^{6}$, equipped with the maximum norm $\|\cdot\|_{\infty}$ and introduce the positive cone

$$
C_{\omega}^{+}:=\left\{\phi \in C_{\omega}: \phi(t) \geq 0, \forall t \in \mathbb{R}\right\}
$$

Following Wang and Zhao (2008), we then define the linear operator $L: C_{\omega} \rightarrow C_{\omega}$ as

$$
(L \phi)(t)=\int_{0}^{\infty} Y(t, t-a) F(t-a) \phi(t-a) d a, \quad \forall t \in \mathbb{R}, \phi \in C_{\omega}
$$

called the next infection operator. The basic reproduction number of (1) is defined as $\mathscr{R}_{0}:=\rho(L)$, i.e. the spectral radius of the next infection operator $L$. 
For the periodic case, let $W(t, \lambda)$ be the monodromy matrix of

$$
\frac{\mathrm{d} \omega}{\mathrm{d} t}=\left(-V(t)+\frac{1}{\lambda} F(t)\right) \omega, \quad t \in \mathbb{R},
$$

with parameter $\lambda \in(0, \infty)$. Since $F(t)$ is non-negative and $-V(t)$ is cooperative, we obtain that $\rho(W(\omega, \lambda))$ is continuous and non-increasing in $\lambda \in(0, \infty)$ and $\lim _{\lambda \rightarrow \infty} \rho(W(\omega, \lambda))<1$.

From the above discussion, we obtain the following result for the local asymptotic stability of the disease free periodic solution $E_{0}$ of our model (1).

Theorem 1 (Wang and Zhao (2008, Theorem 2.1)) The following statements hold.

(i) If $\rho(W(\omega, \lambda))=1$ has a positive solution $\lambda_{0}$, then $\lambda_{0}$ is an eigenvalue of operator $L$, and hence $\mathscr{R}_{0}>0$.

(ii) If $\mathscr{R}_{0}>0$, then $\lambda=\mathscr{R}_{0}$ is the unique solution of $\rho(W(\omega, \lambda))=1$.

(iii) $\mathscr{R}_{0}=0$ if and only if $\rho(W(\omega, \lambda))<1$ for all $\lambda>0$.

Theorem 2 (Wang and Zhao (Wang and Zhao (2008), Theorem 2.2)) The following statements hold.

(i) $\mathscr{R}_{0}=1$ if and only if $\rho\left(\Phi_{F-V}(\omega)\right)=1$;

(ii) $\mathscr{R}_{0}>1$ if and only if $\rho\left(\Phi_{F-V}(\omega)\right)>1$;

(iii) $\mathscr{R}_{0}<1$ if and only if $\rho\left(\Phi_{F-V}(\omega)\right)<1$.

Hence, the disease-free periodic solution $E_{0}$ is locally asymptotically stable if $\mathscr{R}_{0}<1$, and unstable if $\mathscr{R}_{0}>1$.

\subsection{Derivation of the Basic Reproduction Number of the Autonomous Model}

To calculate the basic reproduction ratio $\mathscr{R}_{0}^{A}$ of the autonomous model obtained from (3) by setting the time-dependent parameters (mosquito birth $\left(\tilde{\mu}_{v}(t) \equiv \mu_{v}\right)$ and death rates $\left(\tilde{d}_{v}(t) \equiv d_{v}\right)$ and biting rates $\left(\tilde{\alpha}_{h}(t) \equiv \alpha_{h}\right.$ and $\left.\tilde{\alpha}_{v}(t) \equiv \alpha_{v}\right)$ to constant, we follow the general approach established by Diekmann et al. (2009).

Substituting the values in the disease-free equilibrium $S_{v}^{*}=\frac{\mu_{v}}{d_{v}}$ in equation (7), for all $t \geq 0$, we obtain the Jacobian $F$ given by

$$
F=\left[\begin{array}{cccccc}
\beta \tau_{e} & \beta \tau_{a} & \beta & \beta \tau_{r} & 0 & \alpha_{h} \\
0 & 0 & 0 & 0 & 0 & 0 \\
0 & 0 & 0 & 0 & 0 & 0 \\
0 & 0 & 0 & 0 & 0 & 0 \\
\frac{\eta_{e} \alpha_{v} \mu_{v} d_{h}}{\mu_{h} d_{v}} & \frac{\eta_{a} \alpha_{v} \mu_{v} d_{h}}{\mu_{h} d_{v}} & \frac{\alpha_{v} \mu_{v} d_{h}}{\mu_{h} d_{v}} & 0 & 0 & 0 \\
0 & 0 & 0 & 0 & 0 & 0
\end{array}\right]
$$


and the Jacobian $V$ given by

$$
V=\left[\begin{array}{cccccc}
v_{h}+d_{h} & 0 & 0 & 0 & 0 & 0 \\
-q v_{h} & \gamma_{a}+d_{h} & 0 & 0 & 0 & 0 \\
-(1-q) v_{h} & 0 & \gamma_{s}+d_{h} & 0 & 0 & 0 \\
0 & -\gamma_{a} & -\gamma_{s} & \gamma_{r}+d_{h} & 0 & 0 \\
0 & 0 & 0 & 0 & v_{v}+d_{v} & 0 \\
0 & 0 & 0 & 0 & -v_{v} & d_{v}
\end{array}\right]
$$

therefore the characteristic polynomial of the next generation matrix $F V^{-1}$ is

$$
\lambda^{4}\left(\lambda^{2}-\mathscr{R}_{h h} \lambda-\mathscr{R}_{h v} \mathscr{R}_{v h}\right)=0
$$

where

$$
\begin{aligned}
\mathscr{R}_{h h} & =\frac{\beta}{d_{h}+v_{h}}\left(\tau_{e}+\frac{q \tau_{a} v_{h}}{\gamma_{a}+d_{h}}+\frac{(1-q) v_{h}}{\gamma_{s}+d_{h}}+\frac{\tau_{r} v_{h}\left(\gamma_{s}\left(\gamma_{a}+d_{h}\right)+q\left(\gamma_{a}-\gamma_{s}\right) d_{h}\right)}{\left(\gamma_{a}+d_{h}\right)\left(\gamma_{r}+d_{h}\right)\left(\gamma_{s}+d_{h}\right)}\right), \\
\mathscr{R}_{h v} & =\frac{\alpha_{v} d_{h} \mu_{v}}{d_{v} \mu_{h}\left(d_{h}+v_{h}\right)}\left(\eta_{e}+\frac{q \eta_{a} v_{h}}{\gamma_{a}+d_{h}}+\frac{(1-q) v_{h}}{\gamma_{s}+d_{h}}\right), \\
\mathscr{R}_{v h} & =\frac{\alpha_{h} v_{v}}{d_{v}\left(d_{v}+v_{v}\right)} .
\end{aligned}
$$

The characteristic polynomial therefore is the quadratic equation

$$
\lambda^{2}-\mathscr{R}_{h h} \lambda-\mathscr{R}_{h v} \mathscr{R}_{v h}=0
$$

According to Diekmann et al. (2009), the basic reproduction number is the spectral radius of $F V^{-1}$. Thus, the basic reproduction number corresponds to the dominant eigenvalue given by the root of the quadratic equation (11)

$$
\mathscr{R}_{0}^{A}=\frac{\mathscr{R}_{h h}+\sqrt{\mathscr{R}_{h h}^{2}+4 \mathscr{R}_{h v} R_{v h}}}{2},
$$

where $\mathscr{R}_{h h}$ and $\mathscr{R}_{v}=\mathscr{R}_{h v} \mathscr{R}_{v h}$ are the basic reproduction numbers corresponding to sexual transmission and vector-borne transmission, respectively. From (12), we found that $\mathscr{R}_{h h}+\mathscr{R}_{v}<1$ is the necessary and sufficient condition for $\mathscr{R}_{0}^{A}<1$.

\section{Threshold Dynamics}

Here we study the global stability of the disease-free equilibrium of model (3) and the persistence of the infectious compartments. We use the general theory for the extinction or persistence of infectious given by Rebelo et al. (2011) to show that if the basic reproduction ratio $\mathscr{R}_{0}$ is less than 1 , then the unique disease-free equilibrium $\mathscr{X}^{*}(t)=\left(0,0,0,0,0,0, N_{h}^{*}, N_{h}^{*}, S_{v}^{*}(t)\right)$ is globally asymptotically stable (G.A.S.) 
and the disease dies out, while if the basic reproduction ratio $\mathscr{R}_{0}$ is larger than 1 , the disease persists. Moreover, we follow Zhang and Zhao (2007), Liu et al. (2010), Nakata and Kuniya (2010) and Qu et al. (2017) to prove the existence of a positive periodic solution of (3) if $\mathscr{R}_{0}>1$.

\subsection{Global Stability of the Disease-Free Equilibrium}

In this subsection, we use the general method given by Rebelo et al. (2011) to show that the disease-free equilibrium is G.A.S. if $\mathscr{R}_{0}<1$.

Theorem 3 If $\mathscr{R}_{0}<1$, then the disease-free periodic solution $\mathscr{X}^{*}(t)$ of system (6) is globally asymptotically stable and if $\mathscr{R}_{0}>1$, then it is unstable.

Proof By Theorem 2, we know that $\mathscr{X}^{*}(t)$ is unstable if $\mathscr{R}_{0}>1$ and if $\mathscr{R}_{0}<1$, then $\mathscr{X}^{*}(t)$ is locally asymptotically stable. According to the above discussion in Sect. 3, the conditions (A1) to (A7) in Rebelo et al. (2011) are satisfied.

Moreover $\mathscr{X}^{*}(t)=\left(0,0,0,0,0,0, N_{h}^{*}, N_{h}^{*}, S_{v}^{*}(t)\right)$ is the unique periodic solution in the set of the disease-free states $\mathscr{X}_{s}$.

Clearly, $S(t) \leq N_{h}(t)$, for all $t \geq 0$. From Lemma 1 , for any $\epsilon>0$, there exists $t(\epsilon)>0$ such that $S_{v}(t) \leq N_{v}(t) \leq S_{v}^{*}(t)+\epsilon$ for all $t \geq t(\epsilon)$.

Substituting into system (3), we obtain

$$
\begin{aligned}
& E_{h}^{\prime}(t) \leq \beta\left(\tau_{e} E_{h}(t)+\tau_{a} I_{a}(t)+I_{s}(t)+\tau_{r} I_{r}(t)\right)+\tilde{\alpha}_{h}(t) I_{v}(t)-\left(v_{h}+d_{h}\right) E_{h}(t), \\
& I_{a}^{\prime}(t) \leq q v_{h} E_{h}(t)-\gamma_{a} I_{a}(t)-d_{h} I_{a}(t), \\
& I_{s}^{\prime}(t) \leq(1-q) v_{h} E_{h}(t)-\gamma_{s} I_{s}(t)-d_{h} I_{s}(t), \\
& I_{r}^{\prime}(t) \leq \gamma_{a} I_{a}(t)+\gamma_{s} I_{s}(t)-\gamma_{r} I_{r}(t)-d_{h} I_{r}(t), \\
& E_{v}^{\prime}(t) \leq \tilde{\alpha}_{v}(t) \frac{\eta_{e} E_{h}(t)+\eta_{a} I_{a}(t)+I_{s}(t)}{N_{h}^{*}}\left(S_{v}^{*}(t)+\varepsilon\right)-\left(v_{v}+\tilde{d}_{v}(t)\right) E_{v}(t), \\
& I_{v}^{\prime}(t) \leq v_{v} E_{v}(t)-\tilde{d}_{v}(t) I_{v}(t),
\end{aligned}
$$

for all $t \geq t(\epsilon)$.

Set $\mu(\epsilon):=\min \left\{S_{v}^{*}(\cdot) /\left(S_{v}^{*}(\cdot)+\epsilon\right)\right\}$. Then we have the following system:

$$
\frac{\mathrm{d} \tilde{U}(t)}{\mathrm{d} t} \leq\left(\frac{F(t)}{\mu(\epsilon)}-V(t)\right) \tilde{U}(t), \quad \forall t \geq t(\epsilon)
$$

where $\tilde{U}(t)=\left(\tilde{E}_{h}(t), \tilde{I}_{a}(t), \tilde{I}_{s}(t), \tilde{I}_{r}(t), \tilde{E}_{v}(t), \tilde{I}_{v}(t)\right)$. Then $\tilde{U}(t) \rightarrow 0$ as $t \rightarrow \infty$ and the disease dies out.

By applying the first part of (Rebelo et al. 2011, Theorem 2), we conclude that the disease-free periodic solution $\mathscr{X}^{*}(t)$ is G.A.S. since it is G.A.S. in $\mathscr{X}_{s}$. 


\subsection{Persistence of the Infective Compartments}

In this subsection, we will show that the infectives are persistent if $\mathscr{R}_{0}>1$, by using the general method given by Rebelo et al. (2011).

Theorem 4 If $\mathscr{R}_{0}>1$ then system (3) is persistent with respect to $E_{h}, I_{a}, I_{s}, I_{r}, E_{v}$ and $I_{v}$.

Proof Persistence of $E_{h}+I_{a}+I_{s}$ implies persistence of $E_{h}, I_{a}$ and $I_{s}$, and hence, persistence of $I_{r}, E_{v}$ and $I_{v}$. If there exists $\epsilon>0$ such that $\liminf _{t \rightarrow+\infty}\left(E_{h}+I_{a}+\right.$ $\left.I_{S}\right) \geq \epsilon$, then $E_{h} \geq \frac{\epsilon}{3}-I_{a}-I_{s}$ for large $t$. Thus, from system (3), we obtain

$$
\begin{aligned}
& I_{a}^{\prime} \geq q v_{h} \frac{\epsilon}{3}-\left(q v_{h}+\gamma_{a}+d_{h}\right) I_{a}-q v_{h} I_{s}, \\
& I_{s}^{\prime} \geq(1-q) v_{h} \frac{\epsilon}{3}-\left((1-q) v_{h}+\gamma_{s}+d_{h}\right) I_{s}-(1-q) v_{h} I_{a} .
\end{aligned}
$$

Thus, we have

$$
\begin{aligned}
& I_{a}(t) \geq \frac{\epsilon}{3} \frac{q v_{h}}{q v_{h}+\gamma_{a}+d_{h}}=: \kappa_{a}(\epsilon), \\
& I_{s}(t) \geq \frac{\epsilon}{3} \frac{(1-q) v_{h}}{(1-q) v_{h}+\gamma_{s}+d_{h}}=: \kappa_{s}(\epsilon) .
\end{aligned}
$$

By introducing the inequality (15) into the fifth equation of system (3), we get

$$
I_{r}^{\prime} \geq \gamma_{a} \kappa_{a}(\epsilon)+\gamma_{s} \kappa_{s}(\epsilon)-\left(\gamma_{r}+d_{h}\right) I_{r}
$$

and hence,

$$
I_{r}(t) \geq \frac{\gamma_{a} \kappa_{a}(\epsilon)+\gamma_{s} \kappa_{s}(\epsilon)}{\gamma_{r}+d_{h}}=: \kappa_{r}(\epsilon)
$$

Consider $E_{h} \leq \epsilon, I_{a} \leq \epsilon, I_{s} \leq \epsilon, I_{r} \leq \epsilon, R \leq \epsilon, E_{v} \leq \epsilon$ and $I_{v} \leq \epsilon$ for all $t \geq t_{0}$. There exists $t_{1} \geq t_{0}$ such that $\left|N_{h}(t)-S_{h}^{*}\right| \leq \epsilon$ and $\left|N_{v}(t)-S_{v}^{*}(t)\right| \leq \epsilon$ for all $t \geq t_{1}$. Therefore, $S_{h}(t)=N_{h}(t)-E_{h}(t)-I_{a}(t)-I_{s}(t)-I_{r}(t)-R(t) \geq S_{h}^{*}-5 \epsilon$ and $S_{v}(t)=N_{v}(t)-E_{v}(t)-I_{v}(t) \geq S_{v}^{*}(t)-3 \epsilon$ for all $t \geq t_{1}$. From the equation for $E_{v}^{\prime}$ of system (3), we have

$$
E_{v}^{\prime} \geq \tilde{\alpha}_{v}(t) \frac{\eta_{e} \frac{\epsilon}{3}+\left(\eta_{a}-\eta_{e}\right) I_{a}+\left(1-\eta_{e}\right) I_{s}}{N_{h}^{*}}\left(S_{v}^{*}(t)-3 \epsilon\right)-\left(v_{v}+\tilde{d}_{v}(t)\right) E_{v}
$$

By (Rebelo et al. 2011, Lemma 1), we obtain that

$$
E_{v}(t) \geq \frac{\tilde{\alpha}_{v}^{M}\left(\eta_{e} \frac{\epsilon}{3}+\left(\eta_{a}-\eta_{e}\right) \kappa_{a}(\epsilon)+\left(1-\eta_{e}\right) \kappa_{s}(\epsilon)\right)\left(S_{v}^{* M}(t)-3 \epsilon\right)}{2 N_{h}^{*}\left(v_{v}+\tilde{d}_{v}^{L}\right)}=: \kappa_{e}(\epsilon)
$$


Substituting the inequality (19) into the equation for $I_{v}^{\prime}$ of system (3), we obtain

$$
I_{v}^{\prime} \geq v_{v} \kappa_{e}(\epsilon)-\tilde{d}_{v}(t) I_{v}
$$

and again by (Rebelo et al. 2011, Lemma 1), we have

$$
I_{v}(t) \geq \frac{v_{v} \kappa_{e}(\epsilon)}{2 \tilde{d}_{v}^{L}}=: K_{v}(\epsilon) .
$$

Set $\lambda(\epsilon):=\max \left\{1 /\left(1-\frac{5 \epsilon}{N_{h}^{*}}\right), \max \left(S_{v}^{*}(\cdot) /\left(S_{v}^{*}(\cdot)-3 \epsilon\right)\right)\right\}$. From the equations of system (3), for sufficiently large $t \geq t_{1}$, we obtain

$$
\begin{aligned}
E_{h}^{\prime}(t) & \geq \beta\left(\tau_{e} E_{h}(t)+\tau_{a} I_{a}(t)+I_{s}(t)+\tau_{r} I_{r}(t)+\tilde{\alpha}_{h}(t) I_{v}(t)\right)\left(1-\frac{5 \epsilon}{N_{h}^{*}}\right)-\left(v_{h}+d_{h}\right) E_{h}(t) \\
& \geq \beta\left(\tau_{e} E_{h}(t)+\tau_{a} I_{a}(t)+I_{s}(t)+\tau_{r} I_{r}(t)+\tilde{\alpha}_{h}(t) I_{v}(t)\right) \frac{1}{\lambda(\epsilon)}-\left(v_{h}+d_{h}\right) E_{h}(t), \\
I_{a}^{\prime}(t) & \geq q v_{h} E_{h}(t)-\gamma_{a} I_{a}(t)-d_{h} I_{a}(t), \\
I_{s}^{\prime}(t) & \geq(1-q) v_{h} E_{h}(t)-\gamma_{s} I_{s}(t)-d_{h} I_{s}(t), \\
I_{r}^{\prime}(t) & \geq \gamma_{a} I_{a}(t)+\gamma_{s} I_{s}(t)-\gamma_{r} I_{r}(t)-d_{h} I_{r}(t), \\
E_{v}^{\prime}(t) & \geq \tilde{\alpha}_{v}(t)\left(\eta_{e} E_{h}(t)+\eta_{a} I_{a}(t)+I_{s}(t)\right)\left(\frac{S_{v}^{*}(t)}{N_{h}^{*}}-\frac{3 \epsilon}{N_{h}^{*}}\right)-\left(v_{v}+\tilde{d}_{v}(t)\right) E_{v}(t) \\
& \geq \tilde{\alpha}_{v}(t)\left(\eta_{e} E_{h}(t)+\eta_{a} I_{a}(t)+I_{s}(t)\right) \frac{S_{v}^{*}(t)}{\lambda(\epsilon)}-\left(v_{v}+\tilde{d}_{v}(t)\right) E_{v}(t), \\
I_{v}^{\prime}(t) & \geq v_{v} E_{v}(t)-\tilde{d}_{v}(t) I_{v}(t) .
\end{aligned}
$$

From Lemma 1, it is clear that condition (A8) in Rebelo et al. (2011) is satisfied. Therefore, the assumptions of (Rebelo et al. 2011, Theorem 4) are satisfied and system (3) is persistent with respect to $E_{h}, I_{a}, I_{s}, I_{r}, E_{v}$, and $I_{v}$.

\subsection{Existence of Positive Periodic Solutions}

Define

$$
\begin{aligned}
& X:=\left\{\left(S_{h}, E_{h}, I_{a}, I_{s}, I_{r}, N_{h}, S_{v}, E_{v}, I_{v}\right) \in \mathbb{R}_{+}^{9}\right\}, \\
& X_{0}:=\left\{\left(S_{h}, E_{h}, I_{a}, I_{s}, I_{r}, N_{h}, S_{v}, E_{v}, I_{v}\right) \in \mathbb{R}_{+} \times \operatorname{Int}\left(\mathbb{R}_{+}^{4}\right) \times \mathbb{R}_{+}^{2} \times \operatorname{Int}\left(\mathbb{R}_{+}^{2}\right)\right\},
\end{aligned}
$$

and

$$
\partial X_{0}:=X \backslash X_{0}=\left\{\left(S_{h}, E_{h}, I_{a}, I_{s}, I_{r}, N_{h}, S_{v}, E_{v}, I_{v}\right): E_{h} I_{a} I_{s} I_{r} E_{v} I_{v}=0\right\} .
$$

Let $P: \mathbb{R}_{+}^{9} \rightarrow \mathbb{R}_{+}^{9}$ be the Poincaré map associated with (3), that is,

$$
P\left(x^{0}\right)=u\left(\omega, x^{0}\right), \quad \text { for } x^{0} \in \mathbb{R}_{+}^{9},
$$


where $u\left(t, x^{0}\right)$ is the unique solution of (3) with $u\left(0, x^{0}\right)=x^{0}$. It is easy to see that

$$
P^{m}\left(x^{0}\right)=u\left(m \omega, x^{0}\right), \quad \forall m \geq 0 .
$$

Let $d(x, y)$ denote Euclidean distance in $\mathbb{R}^{9}$. The following lemma is analogous with (Liu et al. 2010, Lemma 3.1).

Lemma 2 If $\mathscr{R}_{0}>1$, then there exists $a \sigma^{*}>0$ such that for any $x^{0} \in X_{0}$, with $\left\|x^{0}-E_{0}\right\| \leq \sigma^{*}$ we have

$$
\limsup _{m \rightarrow \infty} d\left(P^{m}\left(x^{0}\right), E_{0}\right) \geq \sigma^{*}
$$

Proof By Theorem 2, we have that $\rho\left(\Phi_{F-V}(\omega)\right)>1$ if $\mathscr{R}_{0}>1$. Then, we can choose $\eta>0$ small enough such that $\rho\left(\Phi_{F-V-M_{\eta}}(\omega)\right)>1$ where

$$
M_{\eta}(t)=\left[\begin{array}{cccccc}
0 & 0 & 0 & 0 & 0 & 0 \\
0 & 0 & 0 & 0 & 0 & 0 \\
0 & 0 & 0 & 0 & 0 & 0 \\
0 & 0 & 0 & 0 & 0 & 0 \\
\frac{2 \eta}{N_{h}^{*}+\eta} \eta_{e} \tilde{\alpha}_{v}(t) & \frac{2 \eta}{N_{h}^{*}+\eta} \eta_{a} \tilde{\alpha}_{v}(t) \frac{2 \eta}{N_{h}^{*}+\eta} \tilde{\alpha}_{v}(t) & 0 & 0 & 0 \\
0 & 0 & 0 & 0 & 0 & 0
\end{array}\right] .
$$

The equation $\frac{\mathrm{d} S_{h}}{\mathrm{~d} t}=\mu_{h}-d_{h} S_{h}$ has a unique equilibrium $S_{h}^{*}=N_{h}^{*}$ which is a global attractor in $\mathbb{R}_{+}$.

The perturbed system

$$
\frac{\mathrm{d} \hat{S}_{h}(t)}{\mathrm{d} t}=\left(\mu_{h}-\beta \sigma_{1}-\tilde{\alpha}_{h}(t) \sigma_{2}\right)-d_{h} \hat{S}_{h}(t)
$$

has a unique solution

$$
\hat{S_{h}}\left(t, \sigma_{1}, \sigma_{2}\right)=e^{-d_{h} t}\left(\hat{S_{h}}\left(0, \sigma_{1}, \sigma_{2}\right)+\int_{0}^{t} e^{-d_{h} s}\left(\mu_{h}-\beta \sigma_{1}-\alpha_{h}(s) \sigma_{2}\right) \mathrm{d} s\right)
$$

through any initial value $\hat{S_{h}}\left(0, \sigma_{1}, \sigma_{2}\right)$, and it has a unique periodic solution

$$
\hat{S_{h}^{*}}\left(t, \sigma_{1}, \sigma_{2}\right)=e^{-d_{h} t}\left(\hat{S_{h}^{*}}\left(0, \sigma_{1}, \sigma_{2}\right)+\int_{0}^{t} e^{-d_{h} s}\left(\mu_{h}-\beta \sigma_{1}-\alpha_{h}(s) \sigma_{2}\right) \mathrm{d} s\right)
$$

where

$$
\hat{S}_{h}^{*}\left(0, \sigma_{1}, \sigma_{2}\right)=\frac{\int_{0}^{\omega} e^{-d_{h} s}\left(\mu_{h}-\beta \sigma_{1}-\alpha_{h}(s) \sigma_{2}\right) \mathrm{d} s}{e^{-d_{h} \omega}-1}
$$


It is clear that $\left|\hat{S}_{h}\left(t, \sigma_{1}, \sigma_{2}\right)-\hat{S}_{h}^{*}\left(t, \sigma_{1}, \sigma_{2}\right)\right| \rightarrow 0$ as $t \rightarrow \infty$, and from this we obtain that $\hat{S}_{h}^{*}\left(t, \sigma_{1}, \sigma_{2}\right)$ is globally attractive on $\mathbb{R}_{+}$. One can easily see that $\hat{S}_{h}^{*}\left(0, \sigma_{1}, \sigma_{2}\right)$ is continuous in $\sigma_{1}$ and $\sigma_{2}$. As the solution $\hat{S}_{h}^{*}\left(t, \sigma_{1}, \sigma_{2}\right)$ depends continuously on the initial condition and the parameter values, we obtain that $\hat{S}_{h}^{*}\left(t, \sigma_{1}, \sigma_{2}\right)>S_{h}^{*}-\eta$ holds for sufficiently small $\sigma_{1}$ and small $\sigma_{2}$, and all $t \in[0, \omega]$. By the periodicity of $\hat{S}_{h}^{*}\left(t, \sigma_{1}, \sigma_{2}\right)$ and constant $S_{h}^{*}-\eta$, we see that $\hat{S}_{h}^{*}\left(t, \sigma_{1}, \sigma_{2}\right)>S_{h}^{*}-\eta$ holds for sufficiently small $\sigma_{1}$ and small $\sigma_{2}$, and all $t \geq 0$.

Now, let us consider the following perturbed equation

$$
\frac{\mathrm{d} \hat{S_{v}}(t)}{\mathrm{d} t}=\tilde{\mu}_{v}(t)-\left(\tilde{\alpha}_{v}(t) \sigma_{3}+\tilde{d}_{v}(t)\right){\hat{S_{v}}}_{v}(t) .
$$

The Poincaré map associated with (23) has a unique positive fixed point $\hat{S}_{v}^{*}\left(0, \sigma_{3}\right)$ which is globally attractive in $\mathbb{R}_{+}$. Applying the implicit function theorem, we get that $\hat{S_{v}^{*}}\left(0, \sigma_{3}\right)$ is continuous in $\sigma_{3}$. Thus, we further fix $\sigma_{3}>0$ small enough such that

$$
\hat{S}_{v}^{*}\left(t, \sigma_{3}\right)>S_{v}^{*}-\eta
$$

By the continuous dependence of the solutions on the parameters and initial values and by choosing

$$
\sigma:=\min \left\{\sigma_{1}, \sigma_{2}, \sigma_{3}\right\}
$$

there exists a $\sigma^{*}>0$ such that for all $x^{0} \in X_{0}$ with $\left\|x^{0}-E_{0}\right\| \leq \sigma^{*}$, it holds that

$$
\left\|u\left(t, x^{0}\right)-u\left(t, E_{0}\right)\right\| \leq \sigma, \quad \text { for } 0 \leq t \leq \omega .
$$

We further claim that

$$
\limsup _{m \rightarrow \infty} d\left(P^{m}\left(x^{0}\right) \geq \sigma^{*}\right.
$$

Suppose, by contradiction, that (24) does not hold. Then we have

$$
\limsup _{m \rightarrow \infty} d\left(P^{m}\left(x^{0}\right), E_{0}\right)<\sigma^{*}
$$

for some $x^{0} \in X_{0}$. Without loss of generality, we assume that $d\left(P^{m}\left(x^{0}\right), E_{0}\right)<\sigma^{*}$, for all $m \geq 0$. Then, from the above discussion, we have that

$$
\left\|u\left(t, P^{m}\left(x^{0}\right)\right)-u\left(t, E_{0}\right)\right\|<\sigma, \quad \forall m \geq 0, t \in[0, \omega] .
$$

For any $t \geq 0$, let $t=m \omega+t_{1}$, where $t_{1} \in[0, \omega)$ and $m=\left[\frac{t}{\omega}\right]$, which is the greatest integer less than or equal to $\frac{t}{\omega}$. Then, we get

$$
\left\|u\left(t, x^{0}\right)-u\left(t, E_{0}\right)\right\|=\left\|u\left(t_{1}, P^{m}\left(x^{0}\right)\right)-u\left(t_{1}, E_{0}\right)\right\|<\sigma, \quad \forall t \geq 0 .
$$


Set

$$
\left(S_{h}(t), E_{h}(t), I_{a}(t), I_{S}(t), I_{r}(t), N_{h}(t), S_{v}(t), E_{v}(t), I_{v}(t)\right)=u\left(t, x^{0}\right) .
$$

It follows that $E_{h}(t)<\sigma, I_{a}(t)<\sigma, I_{S}(t)<\sigma, I_{r}(t)<\sigma, I_{v}(t)<\sigma$, for all $t \geq 0$ and from system (3), we have

$$
\begin{aligned}
& \frac{d S_{h}(t)}{\mathrm{d} t} \geq\left(\mu_{h}-\beta \sigma-\tilde{\alpha}_{h}(t) \sigma\right)-d_{h} S_{h}(t), \\
& \frac{d S_{v}(t)}{\mathrm{d} t} \geq \tilde{\mu}_{v}(t)-\left(\tilde{\alpha}_{v}(t) \sigma+\tilde{d}_{v}(t)\right) S_{v}(t) .
\end{aligned}
$$

As the periodic solution $\hat{S}_{h}^{*}(t, \sigma)$ of equation (22) is globally attractive on $\mathbb{R}_{+}$and $\hat{S}_{h}^{*}(t, \sigma)>S_{h}^{*}-\eta$, we have

$$
S_{h}(t) \geq S_{h}^{*}-\eta
$$

for $t$ large enough. Also, the fixed point $\hat{S}_{v}^{*}(t, \sigma)$ of the Poincare map corresponding to (23) is globally attractive and $\hat{S_{v}^{*}}(t, \sigma)>S_{v}^{*}(t)-\eta$, there exists a $t$ large enough such that

$$
S_{v}(t, \sigma)>S_{v}^{*}(t)-\eta
$$

From the equations of system (3), for sufficiently large $t$, we obtain

$$
\begin{aligned}
E_{h}^{\prime}(t) \geq & \beta\left(\tau_{e} E_{h}(t)+\tau_{a} I_{a}(t)+I_{s}(t)+\tau_{r} I_{r}(t)\right. \\
& \left.+\tilde{\alpha}_{h}(t) I_{v}(t)\right)\left(1-\frac{2 \eta}{N_{h}^{*}+\eta}\right)-\left(v_{h}+d_{h}\right) E_{h}(t), \\
I_{a}^{\prime}(t)= & q v_{h} E_{h}(t)-\gamma_{a} I_{a}(t)-d_{h} I_{a}(t), \\
I_{s}^{\prime}(t)= & (1-q) v_{h} E_{h}(t)-\gamma_{s} I_{s}(t)-d_{h} I_{s}(t), \\
I_{r}^{\prime}(t)= & \gamma_{a} I_{a}(t)+\gamma_{s} I_{s}(t)-\gamma_{r} I_{r}(t)-d_{h} I_{r}(t), \\
E_{v}^{\prime}(t) \geq & \tilde{\alpha}_{v}(t)\left(\eta_{e} E_{h}(t)+\eta_{a} I_{a}(t)+I_{s}(t)\right)\left(\frac{S_{v}^{*}(t)}{N_{h}^{*}}-\frac{2 \eta}{N_{h}^{*}+\eta}\right)-\left(v_{v}+\tilde{d}_{v}(t)\right) E_{v}(t), \\
I_{v}^{\prime}(t)= & v_{v} E_{v}(t)-\tilde{d}_{v}(t) I_{v}(t) .
\end{aligned}
$$


Next we consider the system

$$
\begin{aligned}
\frac{\mathrm{d} \hat{E}_{h}(t)}{\mathrm{d} t}= & \beta\left(\tau_{e} \hat{E}_{h}(t)+\tau_{a} \hat{I}_{a}(t)+\hat{I}_{s}(t)+\tau_{r} \hat{I}_{r}(t)+\tilde{\alpha}_{h}(t) \hat{I}_{v}(t)\right)\left(1-\frac{2 \eta}{N_{h}^{*}+\eta}\right) \\
& -\left(v_{h}+d_{h}\right) \hat{E}_{h}(t) \\
\frac{\mathrm{d} \hat{I}_{a}(t)}{\mathrm{d} t}= & q v_{h} \hat{E}_{h}(t)-\gamma_{a} \hat{I}_{a}(t)-d_{h} \hat{I}_{a}(t), \\
\frac{\mathrm{d} \hat{I}_{s}(t)}{\mathrm{d} t}= & (1-q) v_{h} \hat{E}_{h}(t)-\gamma_{s} \hat{I}_{s}(t)-d_{h} \hat{I}_{s}(t), \\
\frac{\mathrm{d} \hat{I}_{r}(t)}{\mathrm{d} t}= & \gamma_{a} \hat{I}_{a}(t)+\gamma_{s} \hat{I}_{s}(t)-\gamma_{r} \hat{I}_{r}(t)-d_{h} \hat{I}_{r}(t), \\
\frac{\mathrm{d} \hat{E}_{v}(t)}{\mathrm{d} t}= & \tilde{\alpha}_{v}(t)\left(\eta_{e} \hat{E}_{h}(t)+\eta_{a} \hat{I}_{a}(t)+\hat{I}_{s}(t)\right)\left(\frac{S_{v}^{*}(t)}{N_{h}^{*}}-\frac{2 \eta}{N_{h}^{*}+\eta}\right)-\left(v_{v}+\tilde{d}_{v}(t)\right) \hat{E}_{v}(t), \\
\frac{\mathrm{d} \hat{I}_{v}(t)}{\mathrm{d} t}= & v_{v} \hat{E}_{v}(t)-\tilde{d}_{v}(t) \hat{I}_{v}(t) .
\end{aligned}
$$

Now we have that $\rho\left(\Phi_{F-V-M_{\eta}}(\omega)\right)>1$. Once again by (Zhang and Zhao 2007, Lemma 2.1), there exists a positive, $\omega$-periodic function $p_{2}(t)$ such that $p_{2}(t) \exp \left(\xi_{2} t\right)$ is a solution of (27) and $\xi_{2}=\frac{1}{\omega} \ln \rho\left(\Phi_{F-V+M_{\eta}}(\omega)\right)>0$. For any $J(0) \in \mathbb{R}_{+}^{6}$, we can choose a real number $K_{2}^{*}>0$ such that $J(0) \geq K_{2}^{*} p_{2}(0)$ where

$$
J(t)=\left(E_{h}(t), I_{a}(t), I_{s}(t), I_{r}(t), E_{v}(t), I_{v}(t)\right)^{T}
$$

Applying the comparison principle (Smith and Waltman 1995, Theorem B.1), we obtain $J(t) \geq p_{2}(t) \exp \left(\xi_{2} t\right)$ for all $t>0$, which implies that $\lim _{t \rightarrow \infty} E_{h}(t)=\infty$, $\lim _{t \rightarrow \infty} I_{a}(t)=\infty, \lim _{t \rightarrow \infty} I_{S}(t)=\infty, \lim _{t \rightarrow \infty} I_{r}(t)=\infty, \lim _{t \rightarrow \infty} E_{v}(t)=\infty$ and $\lim _{t \rightarrow \infty} I_{v}(t)=\infty$. This leads to a contradiction, which completes the proof.

Theorem 5 Assume that $\mathscr{R}_{0}>1$. Then system (3) has at least one positive periodic solution, and there exists an $\varepsilon>0$ such that

$$
\begin{aligned}
& \liminf _{t \rightarrow \infty} E_{h}(t) \geq \varepsilon, \quad \liminf _{t \rightarrow \infty} I_{a}(t) \geq \varepsilon, \quad \liminf _{t \rightarrow \infty} I_{S}(t) \geq \varepsilon, \\
& \liminf _{t \rightarrow \infty} I_{r}(t) \geq \varepsilon, \quad \liminf _{t \rightarrow \infty} E_{v}(t) \geq \varepsilon, \quad \liminf _{t \rightarrow \infty} I_{v}(t) \geq \varepsilon,
\end{aligned}
$$

for all $\left(S_{h}(0), E_{h}(0), I_{a}(0), I_{s}(0), I_{r}(0), N_{h}(0), S_{v}(0), E_{v}(0), I_{v}(0)\right) \in X_{0}$

Proof First, we prove that $P$ is uniformly persistent with respect to $\left(X_{0}, \partial X_{0}\right)$, as from this, applying (Zhao 2017, Theorem 3.1.1), it follows that the solution of (3) is uniformly persistent with respect to $\left(X_{0}, \partial X_{0}\right)$.

Let $\phi=\left(S_{h}(0), E_{h}(0), I_{a}(0), I_{s}(0), I_{r}(0), N_{h}(0), S_{v}(0), E_{v}(0), I_{v}(0)\right) \in X_{0}$ be any initial condition. By solving (1) for all $t>0$, we get that

$$
S_{h}(t)=e^{-\int_{0}^{t}\left(a_{h}(s)+d_{h}\right) \mathrm{d} s}\left[S_{h}(0)+\int_{0}^{t} \mu_{h} e^{\int_{0}^{s}\left(a_{h}(r)+d_{h}\right) \mathrm{d} r} \mathrm{~d} s\right]>0,
$$




$$
\begin{aligned}
E_{h}(t) & =e^{-\left(v_{h}+d_{h}\right) t}\left[E_{h}(0)+\int_{0}^{t} a_{h}(s) S_{h}(s) e^{\left(v_{h}+d_{h}\right) s}\right]>0, \\
I_{a}(t) & =e^{-\left(\gamma_{a}+d_{h}\right) t}\left[I_{a}(0)+q v_{h} \int_{0}^{t} E_{h}(s) e^{\left(\gamma_{a}+d_{h}\right) s} \mathrm{~d} s\right]>0, \\
I_{s}(t) & =e^{-\left(\gamma_{s}+d_{h}\right) t}\left[I_{s}(0)+(1-q) v_{h} \int_{0}^{t} E_{h}(s) e^{\left(\gamma_{s}+d_{h}\right) s} \mathrm{~d} s\right]>0, \\
I_{r}(t) & =e^{-\left(\gamma_{r}+d_{h}\right) t}\left[I_{r}(0)+\int_{0}^{t}\left(\gamma_{a} I_{a}(z)+\gamma_{s} I_{s}(z)\right) e^{\left(\gamma_{r}+d_{h}\right) z} \mathrm{~d} z\right]>0 \\
S_{v}(t) & =e^{-\int_{0}^{t}\left(a_{v}(s)+\tilde{d}_{v}(s)\right) \mathrm{d} s}\left[S_{v}(0)+\int_{0}^{t} \tilde{\mu}_{v}(s) e^{\int_{0}^{s}\left(a_{v}(r)+\tilde{d}_{v}(r)\right) \mathrm{d} r} \mathrm{~d} s\right]>0, \\
E_{v}(t) & =e^{-\int_{0}^{t}\left(v_{v}+\tilde{d}_{v}(s)\right) \mathrm{d} s}\left[E_{v}(0)+\int_{0}^{t} a_{v}(s) S_{h}(s) e^{\int_{0}^{s}\left(v_{v}+\tilde{d}_{v}(r)\right) \mathrm{d} r} \mathrm{~d} s\right]>0, \\
I_{v}(t) & =e^{-\int_{0}^{t} \tilde{d}_{v}(s) \mathrm{d} s}\left[I_{v}(0)+v_{v} \int_{0}^{t} E_{v}(s) e^{\int_{0}^{s} \tilde{d}_{v}(r) \mathrm{d} r} \mathrm{~d} s\right]>0,
\end{aligned}
$$

where

$$
\begin{aligned}
& a_{h}(t)=\beta \frac{\tau_{e} E_{h}(t)+\tau_{a} I_{a}(t)+I_{s}(t)+\tau_{r} I_{r}(t)}{N_{h}(t)}+\tilde{\alpha}_{h}(t) \frac{I_{v}(t)}{N_{h}(t)}, \\
& a_{v}(t)=\tilde{\alpha}_{v}(t) \frac{\eta_{e} E_{h}(t)+\eta_{a} I_{a}(t)+I_{s}(t)}{N_{h}(t)} .
\end{aligned}
$$

Hence, we get the positively invariant of $X_{0}$. Since $X$ is also positively invariant and $\partial X_{0}$ is relatively closed in $X$, it gives $\partial X_{0}$ is positively invariant.

Furthermore, from Lemma 1, it follows that system (3) is point dissipative.

Let us introduce

$$
M_{\partial}=\left\{x^{0} \in \partial X_{0}: P^{m}\left(x^{0}\right) \in \partial X_{0}, \forall m \geq 0\right\} .
$$

We will apply the theory of uniform persistence developed in (Zhao 2017) (see also (Zhang and Zhao 2007, Theorem 2.3)). In order to do this, we first show that

$$
M_{\partial}=\left\{\left(S_{h}, 0,0,0,0, N_{h}, S_{v}, 0,0\right): S_{h} \geq 0, N_{h} \geq 0, S_{v} \geq 0\right\} .
$$

Let us note that $M_{\partial} \supseteq\left\{\left(S_{h}, 0,0,0,0, N_{h}, S_{v}, 0,0\right): S_{h} \geq 0, N_{h} \geq 0, S_{v} \geq 0\right\}$. It suffices to prove that

for arbitrary initial condition $x^{0} \in \partial X_{0}, E_{h}(n \omega)=0$ or $I_{a}(n \omega)=0$ or $I_{s}(n \omega)=0$ or $I_{r}(n \omega)=0$ or $E_{v}(n \omega)=0$ or $I_{v}(n \omega)=0$, for all $n \geq 0$.

By contradiction, assume that there exists an $n_{1} \geq 0$ for which

$$
\left(E_{h}\left(n_{1} \omega\right), I_{a}\left(n_{1} \omega\right), I_{s}\left(n_{1} \omega\right), I_{r}\left(n_{1} \omega\right), E_{v}\left(n_{1} \omega\right), I_{v}\left(n_{1} \omega\right)\right)^{T}>0
$$

Thus, (28) implies $N_{h}(t) \geq S_{h}(t)>0, \forall t>n_{1} \omega$. Then, by replacing $t=0$ to $t=n_{1} \omega$ in (28)-(35), we obtain that $S_{h}(t)>0, E_{h}(t)>0, I_{a}(t)>0, I_{S}(t)>0$, $I_{r}(t)>0, N_{h}(t)>0, S_{v}(t)>0, E_{v}(t)>0, I_{v}(t)>0$. This is in contradiction with the positive invariance of $\partial X_{0}$.

By Lemma 2, $P$ is weakly uniformly persistent with respect to $\left(X_{0}, \partial X_{0}\right)$. From Lemma $1, P$ has a global attractor. It follows that $E_{0}$ is an isolated invariant set in $X$ 
and $W^{s}\left(E_{0}\right) \cap X_{0}=\emptyset$. It is clear that every solution in $M_{\partial}$ converges to $E_{0}$ and $E_{0}$ is acyclic in $M_{\partial}$. By (Zhao 2017, Theorem 1.3.1 and Remark 1.3.1), we obtain that $P$ is uniformly persistent with respect to $\left(X_{0}, \partial X_{0}\right)$. Hence, there exists an $\varepsilon>0$ such that

$$
\begin{aligned}
& \liminf _{t \rightarrow \infty} E_{h}(t) \geq \varepsilon, \quad \liminf _{t \rightarrow \infty} I_{a}(t) \geq \varepsilon, \quad \liminf _{t \rightarrow \infty} I_{s}(t) \geq \varepsilon, \\
& \liminf _{t \rightarrow \infty} I_{r}(t) \geq \varepsilon, \quad \liminf _{t \rightarrow \infty} E_{v}(t) \geq \varepsilon, \quad \liminf _{t \rightarrow \infty} I_{v}(t) \geq \varepsilon .
\end{aligned}
$$

By (Zhao (2017), Theorem 1.3.6), $P$ has a fixed point

$$
\bar{\phi}=\left(\bar{S}_{h}(0), \bar{E}_{h}(0), \bar{I}_{a}(0), \bar{I}_{s}(0), \bar{I}_{r}(0), \bar{N}_{h}(0), \bar{S}_{v}(0), \bar{E}_{v}(0), \bar{I}_{v}(0)\right) \in X_{0},
$$

and hence at least one periodic solution $u(t, \bar{\phi})$ of system (3) exists.

Now, we show that $\bar{S}_{h}(0)$ and $\bar{S}_{v}(0)$ are positive. If $\bar{S}_{h}(0)=0=\bar{S}_{v}(0)$, then from (28) and (33) we get that $\bar{S}_{h}(0)>0$ and $\bar{S}_{v}(0)>0$ for all $t>0$. However, using the periodicity of solution, we have $\bar{S}_{h}(0)=\bar{S}_{h}(n \omega)=0$ and $\bar{S}_{v}(0)=\bar{S}_{v}(n \omega)=0$ for all $n \geq 1$, that leads to a contradiction.

\section{Case Study for Ecuador and Colombia: What Changes in the Parameters Might Lead to a Regular Recurrence of Zika Fever?}

In this section, we apply our model to study the spread of Zika in Ecuador during the 2015-17 and in Colombia during the 2015-17 Zika virus epidemic. From Sect. 4, we see that $\mathscr{R}_{0}$ is a threshold parameter for the persistence of the disease in the population (see Theorems 3 and 5). The functions $\tilde{\mu}_{v}(t), \tilde{\alpha}_{h}(t), \tilde{\alpha}_{v}(t)$ and $\tilde{d}_{v}(t)$ are assumed to be time-periodic with one year as a period and, following e.g. Bakary et al. (2018), they are assumed to be of the form $\mu_{v} \cdot\left(\sin \left(\frac{2 \pi}{p} t+b\right)+a\right), \alpha_{h} \cdot\left(\sin \left(\frac{2 \pi}{p} t+b\right)+a\right)$, $\alpha_{v} \cdot\left(\sin \left(\frac{2 \pi}{p} t+b\right)+a\right)$ and $d_{v} \cdot\left(\cos \left(\frac{2 \pi}{p} t+b\right)+a\right)$ where $p$ is period length, $a, b$ are free adjustment parameters and $\mu_{v}, \alpha_{h}, \alpha_{v}, d_{v}$ are the (constant) baseline values of the corresponding time-dependent parameters.

\subsection{Parameter Estimation for Ecuador and Colombia}

To give an estimate for the values of the parameters providing the best fit, we applied Latin Hypercube Sampling, a method used in statistics to assess simultaneous variation of multiple parameters (see, e.g., McKay et al. (1979)).

Figure 2 shows model (1) fitted to data from Ecuador and Colombia (PAHO 2015, 2017). Our model gives a reasonably good fit for both countries, reproducing the single peak of Zika fever in Colombia and the two peaks of Zika fever experienced in Ecuador in two subsequent years. This shows that model (1) is able to reproduce the two types of outcomes of the Zika epidemic observed in South America. We note that in our simulation for Ecuador, before dying out, the epidemic shows a very minor third peak for the following year 2018. This is in accordance with real world data, 


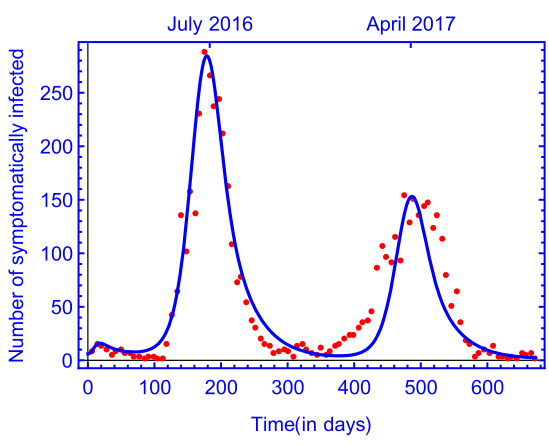

(a) The model fitted to 2016-17 data from Ecuador when $\mathscr{R}_{0}=0.945<1$.

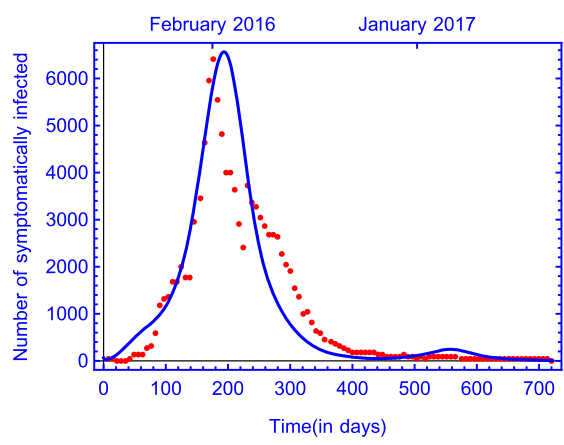

(b) The model fitted to 2015-17 data from colombia when $\mathscr{R}_{0}=0.989<1$.

Fig. 2 (Color figure online) The model fitted to in a 2016-17 data from Ecuador and in b 2015-17 data from Colombia when $\mathscr{R}_{0}<1$ with parameter values in Table 2

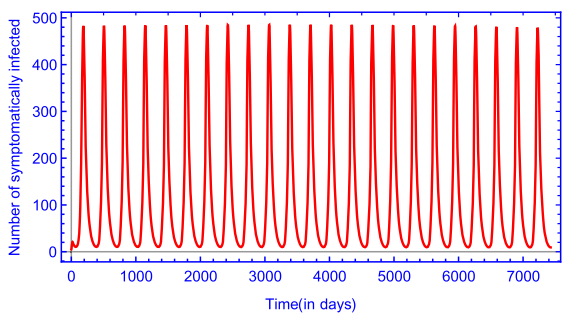

(a) The uniform persistence of the disease in Ecuador when $\alpha_{h}=0.545, \alpha_{v}=0.56, \beta=0.0256$, $\mu_{v}=26,800$ and $\mathscr{R}_{0}=1.2465>1$.

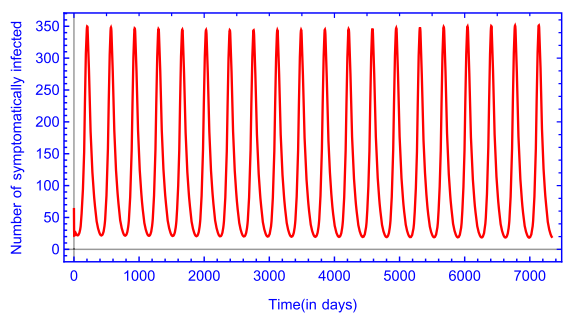

(b) The uniform persistence of the disease in Colombia when $\alpha_{h}=0.641, \alpha_{v}=0.083, \beta=$ $0.0552, \mu_{v}=54,200$ and $\mathscr{R}_{0}=1.707>1$.

Fig. 3 (Color figure online) The uniform persistence of the disease in a Ecuador and in $\mathbf{b}$ Colombia when $\mathscr{R}_{0}>1$. The rest of the parameter values are the same as those in Table 2

as sources report a small number of Zika infections in this year. Our model slightly overestimates the number of cases in 2018, however, as in most cases, Zika fever does not cause severe symptoms and that public awareness was reduced by the decreasing number of Zika cases, probably less people visited their doctors during this third year.

Figure 2 is in accordance with the analytic results stating that the unique disease-free equilibrium $E_{0}$ is globally asymptotically stable when $\mathscr{R}_{0}<1$.

By Theorem 4, system (1) is persistent with respect to the infective compartments if $\mathscr{R}_{0}>1$. Figure 3 shows the persistence of the disease when $\mathscr{R}_{0}>1$.

\subsection{Parameter Changes}

One of our main interests was to see what changes in the parameters might lead to a regular reappearance of the epidemic. Up to now, after 1-3 consecutive years with outbreaks, Zika did not appear in high numbers again. However, in the days of climate change, one can expect that some of the parameters will change in the future as mosquitoes adapt to new circumstances and mutations of the virus appear. This might 
lead to a periodic annual recurrence of the epidemic, just like in the case of other mosquito-borne diseases like dengue fever or malaria. Because of the high number of parameters, it is not easy to assess rigorously which of the parameters have the most important role in the variation of the dynamics, so we only try to demonstrate the possible alterations through a couple of examples.

Our first example (see Fig. 3a) was created with the above determined parameters for the Zika epidemic in Ecuador except the mosquito-related parameters $\alpha_{h}, \alpha_{v}, \beta, \mu_{v}$, i.e. we increased human-to-human and human-to-mosquito transmission rates and mosquito birth rate, while mosquito-to-human transmission rate was decreased. We can calculate numerically the value of the basic reproduction number $\mathscr{R}_{0}=1.2465>1$. In the second example (see Fig. $3 b$ ), similar changes were performed for the parameters determined for Colombia. Again, we can calculate numerically the value of the basic reproduction number $\mathscr{R}_{0}=1.707>1$. Accordingly, one can see that with these parameters, the disease compartments are persistent and the epidemic becomes endemic in the population recurring periodically every year.

In Fig. 4 we present how changes in some of the key parameters (human-to-human, human-to-mosquito and mosquito-to human transmission rates as well as mosquito birth rates) might affect the course of Zika epidemics. The simulations suggest that an increase of any of these four parameters-either due to climate change or to genetic mutation of the virus - can lead to a periodic annual reappearance of the epidemic.

Knowing the seasonal fluctuation, one may rightly suppose that mosquito control is limited to the peak months of mosquito abundance. Hence, we also show an example where mosquito control only occurs during 5 months when the highest number of vectors are present to see whether control measures implemented only during a limited period of the year might have a sufficient effect to eradicate the disease. Mosquito killing is incorporated into the model by considering a step function multiplier of the mosquitoes' death rate. Namely, we increase the death rate during a five-month-long period of each year. For a better assessment of the effect of additional mosquito killing, we assume a periodic recurrence of the disease, just like given in Fig. 3 and Table 2. In Fig. 5 we show some seasonal measures to control Zika virus disease both in Ecuador and Colombia. The figure suggests that even a mosquito control limited to the peak period of mosquito abundance might have a significant impact to control the disease.

\subsection{Sensitivity Analysis}

Sensitivity analysis, using Partial Rank Correlation Coefficients (PRCC, see, e.g., Blower and Dowlatabadi (1994)), is carried out to determine the parameters that have the greatest influence on the dynamics of the diseases. The PRCC-based sensitivity analysis measures the effect of the parameters on the response function (in our cases, the number of infected cases), while we vary the parameters (relevant to the dynamics of the diseases in Ecuador and Colombia) in the given ranges (see Table 2).

Figure 6 shows the comparison of the PRCC values obtained for the parameters $\beta, \alpha_{h}, \alpha_{v}, \mu_{v}$ and $d_{v}$, i.e., those parameters which can typically be affected by control measures. The results suggest that the most relevant factors in Zika transmission, and 

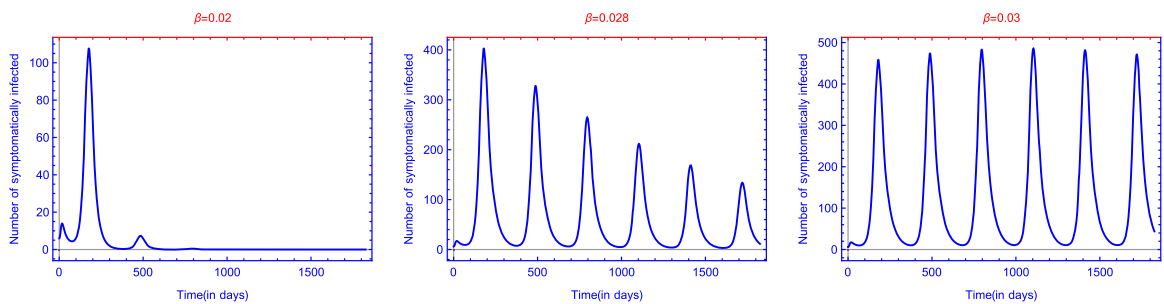

(a) The model solution with different values of $\beta$.
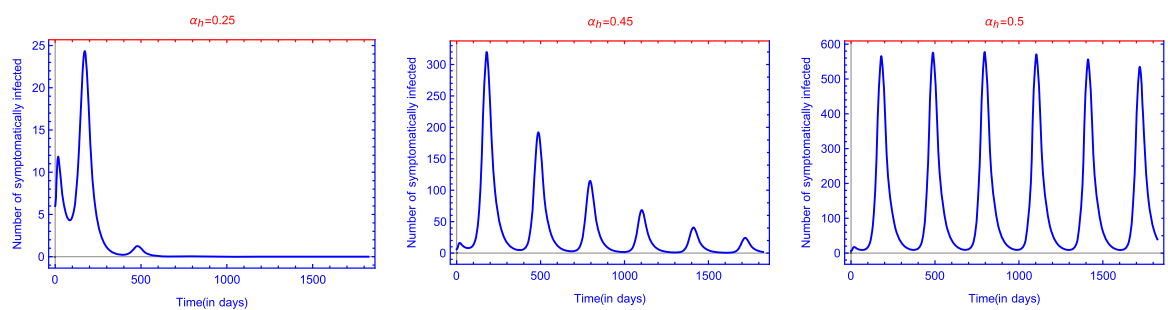

(b) The solution of model (1) with three different values of $\alpha_{h}$.
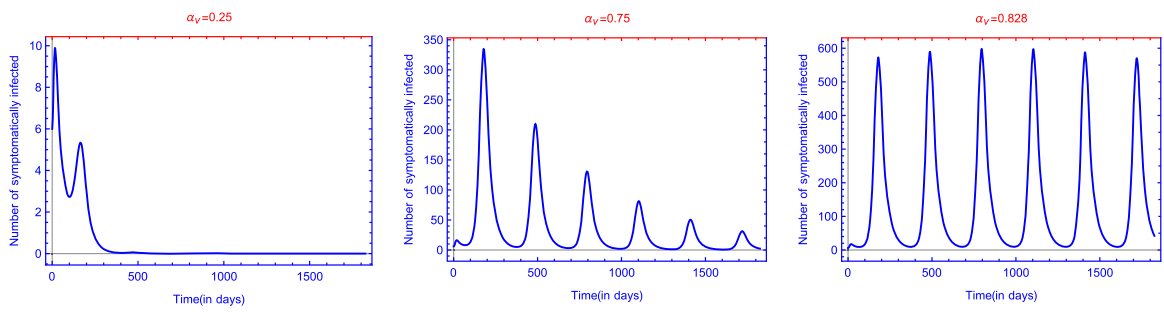

(c) The solution of model (1) with three different values of $\alpha_{v}$.
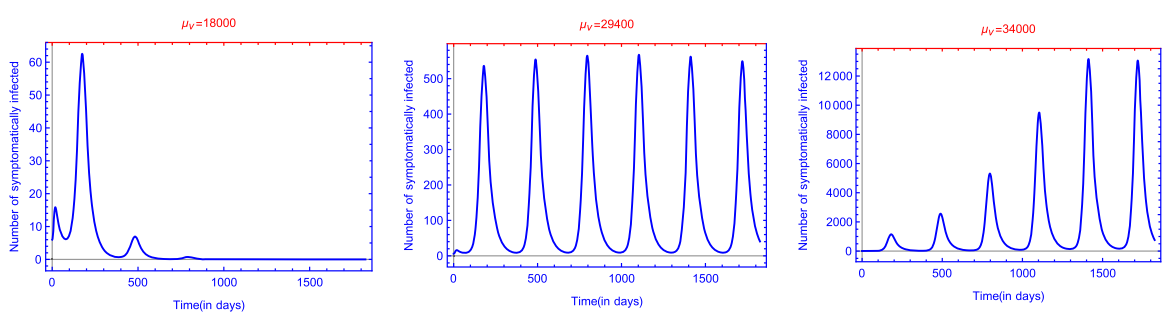

(d) The solution of model (1) with three values of $\mu_{v}$.

Fig. 4 (Color figure online) The solution of model (1) with three different values of in a human-to-human transmission rate $(\beta)$, in $\mathbf{b}$ baseline value of mosquito-to-human transmission rate $\left(\alpha_{h}\right)$, in $\mathbf{c}$ baseline value of human-to-mosquito transmission rate $\left(\alpha_{v}\right)$ and in $\mathbf{d}$ baseline value of mosquito birth rate $\left(\mu_{v}\right)$. The rest of the parameter values are the same as those for Ecuador in Table 2

hence in the elevation of the number of infected cases are birth and death rates of mosquitoes. Spread via sexual contacts is shown to have a smaller effect; however, it is still an important factor. Based on the sensitivity analysis, we can assess that the most effective measures to reduce transmission are control of mosquito populations and protection against their bites. 


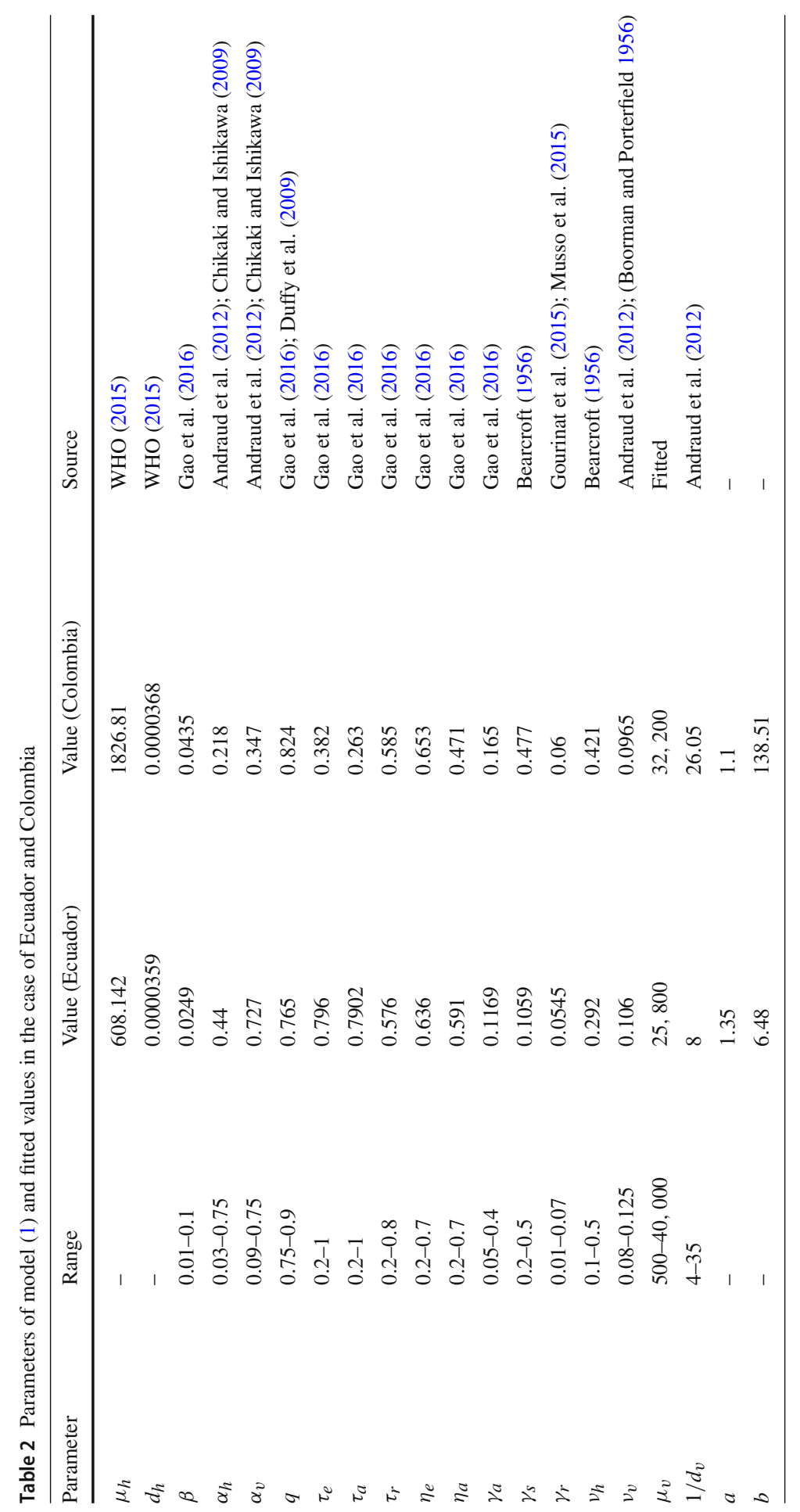




\subsection{Reproduction Numbers}

To calculate the reproduction numbers, we use the parameters as obtained in the fitting to Ecuador data in Table 2. Formula (12) provides us the basic reproduction number in any time point by substituting the parameter values. Figure 7 shows the basic reproduction number of the time-constant model w.r.t. baseline value of mosquito birth rate, baseline value of human-to-mosquito transmission rates and human-tohuman transmission rate, suggesting that control of mosquito population and sexual protection both have a significant effect in Zika fever transmission. The results also imply that vector control might not be enough to contain the disease spread in case of a high sexual transmission rate.

Further, by numerical calculations we get the curves of the basic reproduction ratio $\mathscr{R}_{0}$, the time-average basic reproduction number $\left[\mathscr{R}_{0}\right]$ (using the notation presented by Mitchell and Kribs (2017)) and the basic reproduction number $\mathscr{R}_{0}^{A}$ of the autonomous model with respect to baseline value of mosquito birth rate $\left(\mu_{v}\right)$, human-to-human transmission rate $(\beta)$, baseline value of mosquito-to-human transmission rate $\left(\alpha_{h}\right)$ and baseline value of human-to-mosquito transmission rate $\left(\alpha_{v}\right)$, respectively, in Fig. 8.

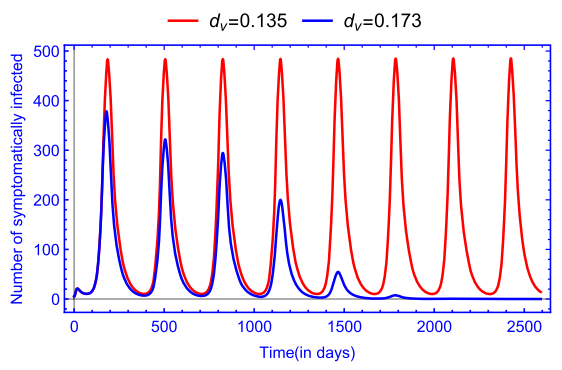

(a) Ecuador.

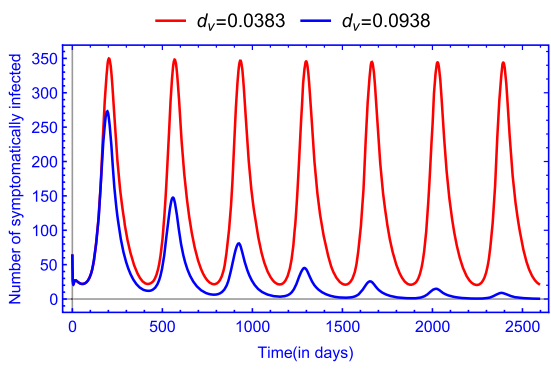

(b) Colombia.

Fig. 5 (Color figure online) Seasonal measures to control ZIKV in a Ecuador and in $\mathbf{b}$ Colombia. The rest of the parameter values are the same as those in Fig. 3 and Table 2

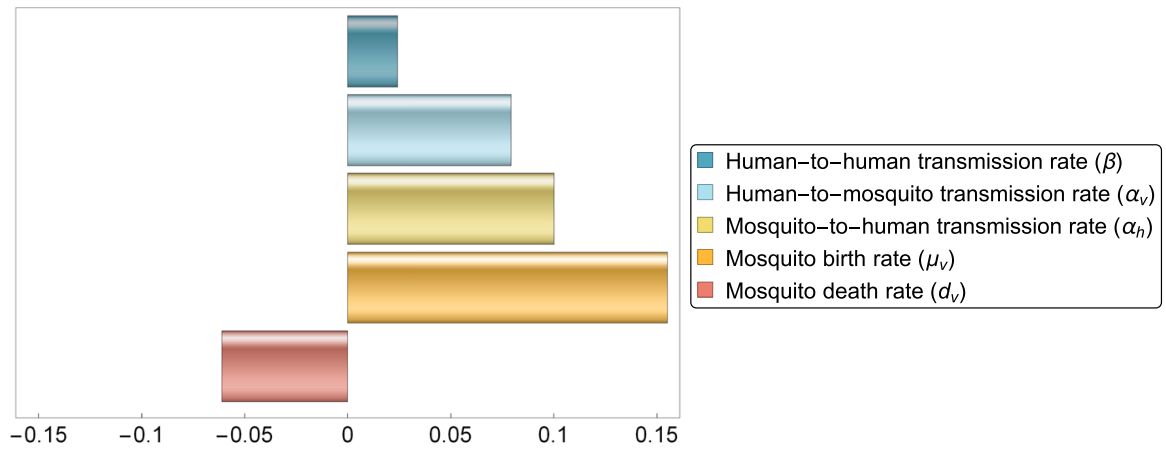

Fig. 6 (Color figure online) Partial rank correlation coefficients of the five parameters subject to intervention measures. Parameters with positive PRCC values are positively correlated with the cumulative number of cases. Parameters with negative PRCC values are negatively correlated with the cumulative number of infections 

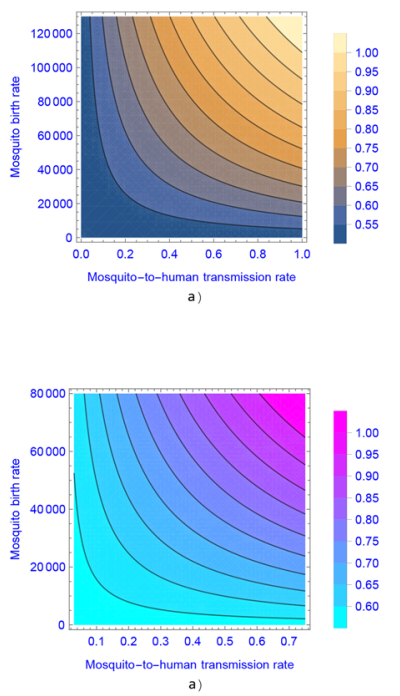
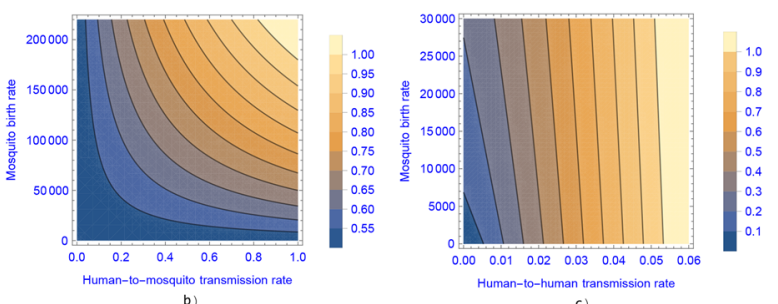

(a) Ecuador

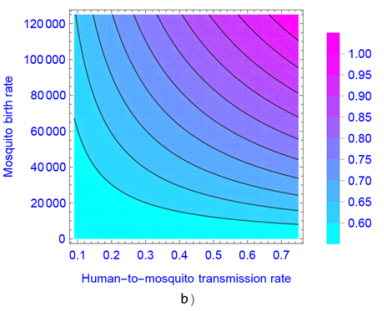

(b) Colombia

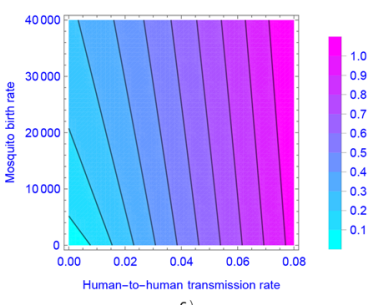

c)

Fig. 7 (Color figure online) Contour plot of the basic reproduction number as a function of baseline value of mosquito birth rate $\left(\mu_{v}\right)$ and a baseline value of mosquito-to-human transmission rate $\left(\alpha_{h}\right)$, b baseline value of human-to-mosquito transmission rate $\left(\alpha_{v}\right)$ and $\mathbf{c}$ human-to-human transmission rate $(\beta)$

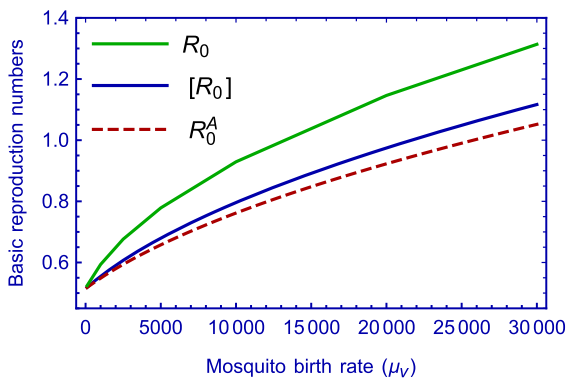

(a)

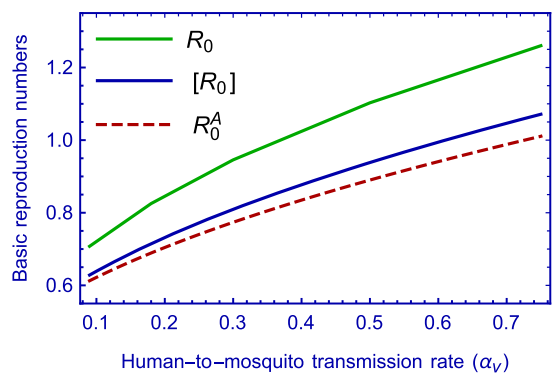

(c)

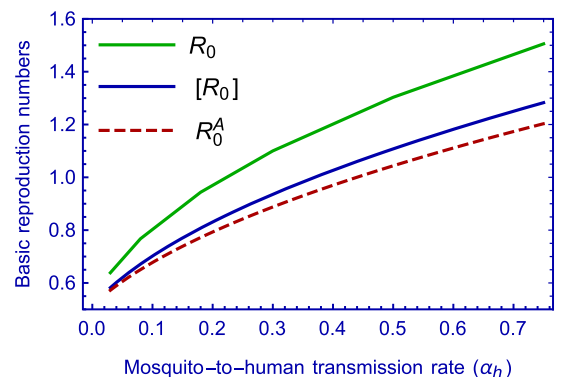

(b)

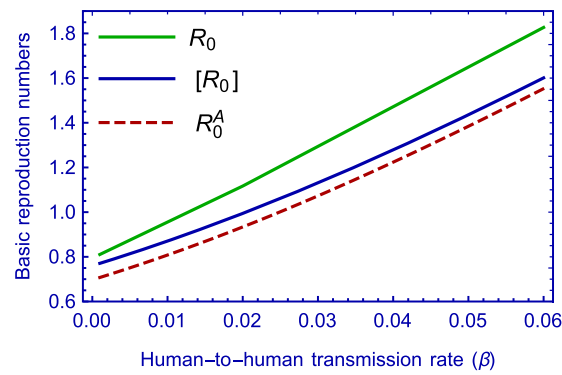

(d)

Fig. 8 (Color figure online) The curves of the basic reproduction number $\mathscr{R}_{0}$, the time-average basic reproduction number $\left[\mathscr{R}_{0}\right]$ and the basic reproduction number of the autonomous model $\mathscr{R}_{0}^{A}$ versus a baseline value of mosquito birth rate $\left(\mu_{v}\right)$, b baseline value of mosquito-to-human transmission rate $\left(\alpha_{h}\right), \mathbf{c}$ baseline value of human-to-mosquito transmission rate $\left(\alpha_{v}\right)$ and $\mathbf{d}$ human-to-human transmission rate $(\beta)$ 
The calculations show that the time-average basic reproduction number $\left[\mathscr{R}_{0}\right]$ is always less than the basic reproduction ratio $\mathscr{R}_{0}$, suggesting that the time-average basic reproduction number underestimates the disease transmission risk. From this aspect, our results are similar to those of Wang and Zhao (2008). We note that there are some other cases of underestimation and overestimation for the average basic reproduction number can be found in (Bacaër 2007), where an approximate formula of the basic reproduction number was obtained for a class of periodic vector-borne disease models with a small perturbation parameter.

\section{Discussion}

We have developed a compartmental population model to describe the transmission of Zika virus disease in a periodic environment (by including periodic coefficients). We have shown that the global dynamics of the model is determined by the basic reproduction number $\mathscr{R}_{0}$. For $\mathscr{R}_{0}$ less than 1 , we have shown the global asymptotic stability of the disease-free periodic solution $E_{0}$, while the disease persists if $\mathscr{R}_{0}>1$. Using our model and taking Ecuador and Colombia as two examples, the fitted curves match the data very well (see Fig. 2). Our numerical simulations suggest that there exists a single positive periodic solution which is globally asymptotically stable for $\mathscr{R}_{0}>1$ (see Fig. 3).

The reproduction numbers were calculated as a function of the parameters $\mu_{v}, \alpha_{h}$, $\alpha_{v}$ and $\beta$. As is observed, the time-average basic reproduction number [ $\left.\mathscr{R}_{0}\right]$ is always less than the basic reproduction number $\mathscr{R}_{0}$ (see Fig. 8). This implies that the timeaverage basic reproduction number underrates the risk of disease transmission, while the risk of infection is overestimated by the basic reproduction number.

Although a regular periodic recurrence of Zika has not been observed so far, it is expected that this might be altered by climate change. Our model allows us to estimate what kind of parameter changes might lead to a periodic recurrence of Zika. Using numerical simulations, we found that mosquito birth and death rates are the most significant factors in a possible periodic recurrence of Zika, however, sexual transmission also has a significant effect on the prevalence of the disease.

Acknowledgements M. A. Ibrahim was supported by Stipendium Hungaricum scholarship with Application No. 173177 and by a fellowship from the Egyptian government in the long-term mission system. A. Dénes was supported by the János Bolyai Research Scholarship of the Hungarian Academy of Sciences, by the Projects Nos. 128363 and 124016, implemented with the support provided from the National Research, Development and Innovation Fund of Hungary, financed under the PD_18 and FK_17 funding schemes, respectively. The research was supported by the Project TUDFO/47138-1/2019-ITM.

Funding Open Access funding provided by University of Szeged.

Open Access This article is licensed under a Creative Commons Attribution 4.0 International License, which permits use, sharing, adaptation, distribution and reproduction in any medium or format, as long as you give appropriate credit to the original author(s) and the source, provide a link to the Creative Commons licence, and indicate if changes were made. The images or other third party material in this article are included in the article's Creative Commons licence, unless indicated otherwise in a credit line to the material. If material is not included in the article's Creative Commons licence and your intended use is not permitted 
by statutory regulation or exceeds the permitted use, you will need to obtain permission directly from the copyright holder. To view a copy of this licence, visit http://creativecommons.org/licenses/by/4.0/.

\section{References}

Andraud M, Hens N, Marais C, Beutels P (2012) Dynamic epidemiological models for dengue transmission: a systematic review of structural approaches. PLoS ONE 7(11):e49085. https://doi.org/10.1371/ journal.pone.0049085

Baca-Carrasco D, Velasco-Hernández JX (2016) Sex, mosquitoes and epidemics: an evaluation of zika disease dynamics. Bull Math Biol 78(11):2228-2242. https://doi.org/10.1007/s11538-016-0219-4

Bacaër N (2007) Approximation of the basic reproduction number $R_{0}$ for vector-borne diseases with a periodic vector population. Bull Math Biol 69(3):1067-1091. https://doi.org/10.1007/s11538-0069166-9

Bacaër N, Ait Dads EH (2011) On the biological interpretation of a definition for the parameter $R_{0}$ in periodic population models. J Math Biol 65(4):601-621. https://doi.org/10.1007/s00285-011-0479-4

Bacaër N, Guernaoui S (2006) The epidemic threshold of vector-borne diseases with seasonality. J Math Biol 53(3):421-436. https://doi.org/10.1007/s00285-006-0015-0

Bakary T, Boureima S, Sado T (2018) A mathematical model of malaria transmission in a periodic environment. J Biol Dyn 12(1):400-432. https://doi.org/10.1080/17513758.2018.1468935

Bearcroft W (1956) Zika virus infection experimentally induced in a human volunteer. Trans R Soc Trop Med Hyg 50(5):438-441. https://doi.org/10.1016/0035-9203(56)90090-6

Blower SM, Dowlatabadi H (1994) Sensitivity and uncertainty analysis of complex models of disease transmission: an HIV model, as an example. Int Stat Rev 62(2):229. https://doi.org/10.2307/1403510

Boorman J, Porterfield J (1956) A simple technique for infection of mosquitoes with viruses transmission of Zika virus. Trans R Soc Trop Med Hyg 50(3):238-242. https://doi.org/10.1016/0035-9203(56)900293

Brauer F, Castillo-Chavez C, Mubayi A, Towers S (2016) Some models for epidemics of vector-transmitted diseases. Inf Dis Model 1(1):79-87. https://doi.org/10.1016/j.idm.2016.08.001

Caminade C, Turner J, Metelmann S, Hesson JC, Blagrove MSC, Solomon T, Morse AP, Baylis M (2016) Global risk model for vector-borne transmission of Zika virus reveals the role of El Niño 2015. Proc Nat Acad Sci 114(1):119-124. https://doi.org/10.1073/pnas.1614303114

Chikaki E, Ishikawa H (2009) A dengue transmission model in Thailand considering sequential infections with all four serotypes. J Infect Dev Ctries. https://doi.org/10.3855/jidc.616

Dénes A, Ibrahim MA, Oluoch L, Tekeli M, Tekeli T (2019) Impact of weather seasonality and sexual transmission on the spread of Zika fever. Sci Rep. https://doi.org/10.1038/s41598-019-53062-z

Dick G, Kitchen S, Haddow A (1952) Zika virus Isolations and serological specificity. Trans R Soc Trop Med Hyg 46(5):509-520. https://doi.org/10.1016/0035-9203(52)90042-4

Diekmann O, Heesterbeek JAP, Roberts MG (2009) The construction of next-generation matrices for compartmental epidemic models. J R Soc Interface 7(47):873-885. https://doi.org/10.1098/rsif.2009.0386

Duffy MR, Chen TH, Hancock WT, Powers AM, Kool JL, Lanciotti RS, Pretrick M, Marfel M, Holzbauer S, Dubray C, Guillaumot L, Griggs A, Bel M, Lambert AJ, Laven J, Kosoy O, Panella A, Biggerstaff BJ, Fischer M, Hayes EB (2009) Zika virus outbreak on Yap Island, Federated States of Micronesia. N Engl J Med 360(24):2536-2543. https://doi.org/10.1056/nejmoa0805715

Gao D, Lou Y, He D, Porco TC, Kuang Y, Chowell G, Ruan S (2016) Prevention and control of Zika as a mosquito-borne and sexually transmitted disease: a mathematical modeling analysis. Sci Rep. https:// doi.org/10.1038/srep28070

Gourinat AC, O'Connor O, Calvez E, Goarant C, Dupont-Rouzeyrol M (2015) Detection of Zika virus in urine. Emerg Infect Dis 21(1):84-86. https://doi.org/10.3201/eid2101.140894

Ibrahim MA, Dénes A (2019) Assessment of microcephaly risk due to Zika virus infection via a mathematical model with vertical transmission, under review

Liu L, Zhao XQ, Zhou Y (2010) A tuberculosis model with seasonality. Bull Math Biol 72(4):931-952. https://doi.org/10.1007/s11538-009-9477-8

Magalhaes T, Foy BD, Marques ET, Ebel GD, Weger-Lucarelli J (2018) Mosquito-borne and sexual transmission of Zika virus: recent developments and future directions. Virus Res 254:1-9. https://doi.org/ 10.1016/j.virusres.2017.07.011 
McKay MD, Beckman RJ, Conover WJ (1979) A comparison of three methods for selecting values of input variables in the analysis of output from a computer code. Technometrics 21(2):239-245. https://doi. org/10.2307/1268522

Mead PS, Duggal NK, Hook SA, Delorey M, Fischer M, McGuire DO, Becksted H, Max RJ, Anishchenko M, Schwartz AM, Tzeng WP, Nelson CA, McDonald EM, Brooks JT, Brault AC, Hinckley AF (2018) Zika virus shedding in semen of symptomatic infected men. N Engl J Med 378(15):1377-1385. https:// doi.org/10.1056/nejmoa1711038

Mitchell C, Kribs C (2017) A comparison of methods for calculating the basic reproductive number for periodic epidemic systems. Bull Math Biol 79(8):1846-1869. https://doi.org/10.1007/s11538-0170309-y

Mordecai EA, Cohen JM, Evans MV, Gudapati P, Johnson LR, Lippi CA, Miazgowicz K, Murdock CC, Rohr JR, Ryan SJ, Savage V, Shocket MS, Ibarra AS, Thomas MB, Weikel DP (2017) Detecting the impact of temperature on transmission of Zika, dengue, and chikungunya using mechanistic models. PLOS Negl Trop Dis 11(4):e0005568. https://doi.org/10.1371/journal.pntd.0005568

Musso D, Roche C, Robin E, Nhan T, Teissier A, Cao-Lormeau VM (2015) Potential sexual transmission of Zika virus. Emerg Infect Dis 21(2):359-361. https://doi.org/10.3201/eid2102.141363

Nakata Y, Kuniya T (2010) Global dynamics of a class of SEIRS epidemic models in a periodic environment. J Math Anal Appl 363(1):230-237. https://doi.org/10.1016/j.jmaa.2009.08.027

Padmanabhan P, Seshaiyer P, Castillo-Chavez C (2017) Mathematical modeling, analysis and simulation of the spread of Zika with influence of sexual transmission and preventive measures. Lett Biomath. https://doi.org/10.30707/lib4.1padmanabhan

Pan American Health Organization (2015) Countries and territories with autochthonous transmission of Zika virus in the Americas reported in 2015-2017. https://www.paho.org/hq/index.php?option=com_ content \&view=article \&id=11603: countries-and-territories- with-autochthonous-transmission-ofzika-virus-in-the-americas-reported-in-2015-2017\&Itemid=41696\&lang=en

Pan American Health Organization (2017) Zika-Epidemiological Report Colombia. https://www.paho.org/ hq/dmdocuments/2017/2017-phe-zika-situation-report-col.pdf

Petersen LR, Jamieson DJ, Powers AM, Honein MA (2016) Zika virus. N Engl J Med 374(16):1552-1563. https://doi.org/10.1056/nejmra1602113

Qu Q, Fang C, Zhang L, Jia W, Weng J, Li Y (2017) A mumps model with seasonality in China. Inf Dis Model 2(1):1-11. https://doi.org/10.1016/j.idm.2016.10.001

Rebelo C, Margheri A, Bacar N (2011) Persistence in seasonally forced epidemiological models. J Math Biol 64(6):933-949. https://doi.org/10.1007/s00285-011-0440-6

Sasmal SK, Ghosh I, Huppert A, Chattopadhyay J (2018) Modeling the spread of Zika virus in a stagestructured population: effect of sexual transmission. Bull Math Biol 80(11):3038-3067. https://doi. org/10.1007/s11538-018-0510-7

Smith HL, Waltman P (1995) The theory of the chemostat, Cambridge Studies in Mathematical Biology, vol 13. Cambridge University Press, Cambridge, dynamics of microbial competition. https://doi.org/ 10.1017/CBO9780511530043

Smithburne KC (1952) Neutralizing antibodies against certain recently isolated viruses in the sera of human beings residing in East Africa. J Immunol 69(2):223-234

Wang L, Teng Z, Zhang T (2013) Threshold dynamics of a malaria transmission model in periodic environment. Commun Nonlinear Sci Numer Simul 18(5):1288-1303. https://doi.org/10.1016/j.cnsns.2012. 09.007

Wang W, Zhao XQ (2008) Threshold dynamics for compartmental epidemic models in periodic environments. J Dyn Differ Equ 20(3):699-717. https://doi.org/10.1007/s10884-008-9111-8

World Health Organization (2015) WHO Global Health Observatory data repository. Crude birth and death rate, Data by country http://apps.who.int/gho/data/node.main.CBDR107?lang=en

World Health Organization (2018) Zika virus. https://www.who.int/en/news-room/fact-sheets/detail/zikavirus

Zhang F, Zhao XQ (2007) A periodic epidemic model in a patchy environment. J Math Anal Appl 325(1):496-516. https://doi.org/10.1016/j.jmaa.2006.01.085

Zhao XQ (2017) Dynamical systems in population biology, 2nd edn. CMS Books in Mathematics. Springer, Cham, https://doi.org/10.1007/978-3-319-56433-3

Publisher's Note Springer Nature remains neutral with regard to jurisdictional claims in published maps and institutional affiliations. 